\title{
ASYMPTOTIC BEHAVIOR OF SOLUTIONS OF A FREE BOUNDARY PROBLEM MODELING THE GROWTH OF TUMORS WITH FLUID-LIKE TISSUE UNDER THE ACTION OF INHIBITORS
}

\author{
JUNDE WU AND FUJUN ZHOU
}

\begin{abstract}
In this paper we study a free boundary problem modeling the growth of tumors with fluid-like tissue under the action of inhibitors. The model includes two elliptic equations describing the concentration of nutrients and inhibitors, respectively, and a Stokes equation for the fluid velocity and internal pressure. By employing the functional approach, analytic semigroup theory and Cui's local phase theorem for parabolic differential equations with invariance, we prove that if a radial stationary solution is asymptotically stable under radial perturbations, then there exists a non-negative threshold value $\gamma_{*}$ such that if $\gamma>\gamma_{*}$, then it keeps asymptotically stable under non-radial perturbations. While if $0<\gamma<\gamma_{*}$, then the radial stationary solution is unstable and, in particular, there exists a center-stable manifold such that if the transient solution exists globally and is contained in a sufficiently small neighborhood of the radial stationary solution, then it converges exponentially to this radial stationary solution (modulo translations) and its translation lies on the center-stable manifold. The result indicates an interesting phenomenon that an increasing inhibitor uptake has a positive effect on the tumor's treatment and can promote the tumor's stability.
\end{abstract}

\section{INTRODUCTION}

During the past several decades, considerable attention has been attracted to mathematical models for the growth of various tumors in vivo and in vitro. Most models are considered solid tumors and it is assumed that the tumor tissue has the structure of porous medium so that Darcy's law applies (see, e.g., [2]- 5], 22, 24] and the references cited therein). Accordingly, many significant results have been obtained for rigorous analysis of such solid tumor models (cf. [2], 6]-[11, 20], [21], 27], 31]). However, there exist tumors for which the tissue structure is more naturally modeled as fluid. For example, in early stages of ductal carcinoma in situ of the breast, the tumor is confined to the duct of a mammary gland, which consists of epithelial cells, a meshwork of proteins and extracellular fluid. In modeling, this leads to the employment of the Stokes equation rather than Darcy's law to model

Received by the editors May 23, 2011 and, in revised form, November 18, 2011.

2010 Mathematics Subject Classification. Primary 35B40, 35R35; Secondary 35Q92, 92C37.

Key words and phrases. Free boundary problem, tumor growth, Stokes equation, radial stationary solution, asymptotic stability.

This work was supported by the National Natural Science Foundation of China under the grant numbers 10901057 and 11001192, the Doctoral Foundation of Education Ministry of China under the grant numbers 200805611027 and 20103201120017, the Natural Science Fund for Colleges and Universities in Jiangsu Province under the grant number 10KJB110008, and the Fundamental Research Funds for the Central Universities of SCUT under the grant number 2012ZZ0072. 
the tumor growth [12-17]. In this paper we study such mathematical models for tumors with fluid-like tissue under the action of inhibitors:

$$
\begin{gathered}
\Delta \sigma=\lambda_{1} \sigma+\beta \quad \text { in } \Omega(t), t>0, \\
\Delta \beta=\lambda_{2} \beta \quad \text { in } \Omega(t), t>0, \\
\sigma=\bar{\sigma} \quad \text { on } \partial \Omega(t), \quad t>0, \\
\beta=\bar{\beta} \quad \text { on } \partial \Omega(t), \quad t>0, \\
\nabla \cdot \mathbf{v}=\mu(\sigma-\hat{\sigma})-\nu \beta \quad \text { in } \Omega(t), t>0, \\
-\Delta \mathbf{v}+\nabla p-\frac{1}{3} \nabla(\nabla \cdot \mathbf{v})=0 \quad \text { in } \Omega(t), t>0, \\
\mathbf{T}(\mathbf{v}, p) \mathbf{n}=-\gamma \kappa \mathbf{n} \quad \text { on } \partial \Omega(t), t>0, \\
V_{\mathbf{n}}=\mathbf{v} \cdot \mathbf{n} \quad \text { on } \partial \Omega(t), t>0, \\
\int_{\Omega(t)} \mathbf{v} \mathrm{d} x=0, \quad t>0, \\
\int_{\Omega(t)}^{\mathbf{v} \times x \mathrm{~d} x=0,} \quad t>0, \\
\Omega(0)=\Omega_{0},
\end{gathered}
$$

where $\sigma=\sigma(t, x), \beta=\beta(t, x), \mathbf{v}=\mathbf{v}(t, x)\left(=\left(v_{1}(t, x), v_{2}(t, x), v_{3}(t, x)\right)\right)$ and $p=p(t, x)$ are unknown functions representing the nutrient concentration, the inhibitor concentration, the fluid velocity and the fluid pressure, respectively, and $\Omega(t)$ is an a priori unknown bounded domain in $\mathbb{R}^{3}$ occupied by the tumor at time $t$. $\lambda_{1}, \lambda_{2}, \mu, \nu, \bar{\sigma}, \bar{\beta}, \hat{\sigma}$ and $\gamma$ are positive constants, among which $\lambda_{1}$ and $\lambda_{2}$ denote the nutrient and inhibitor consumption rate, respectively, $\mu$ is the proliferation rate, $\nu$ reflects the negative function of the inhibitor on the tumor's growth, $\bar{\sigma}$ and $\bar{\beta}$ mean the constant nutrient and inhibitor supply that the tumor receives from external host tissue, $\hat{\sigma}$ is the threshold nutrient concentration for apoptosis, and $\gamma$ denotes the surface tension coefficient of the tumor surface. Besides, $\kappa, \mathbf{n}$ and $V_{\mathbf{n}}$ are the mean curvature, the unit outward normal and the normal velocity, respectively, of the free boundary $\partial \Omega(t)$. The sign of $\kappa$ is defined by the convention that $\kappa \geq 0$ at points of convex hypersurfaces. $\mathbf{T}(\mathbf{v}, p)$ is the stress tensor given by

$$
\mathbf{T}(\mathbf{v}, p)=\left[\nabla \otimes \mathbf{v}+(\nabla \otimes \mathbf{v})^{T}\right]-\left(p+\frac{2}{3} \nabla \cdot \mathbf{v}\right) \mathbf{I},
$$

where $\mathbf{I}$ denotes the unit tensor.

The problem (1.1)-(1.11) was first originated by Franks et al. in [12]-15] and concisely reformulated by Friedman in [16, 17. As an extension, the action of inhibitors on the tumor's growth is also considered in this paper. In this model, the stationary diffusion equations (1.1) and (1.2) combining with the boundary conditions (1.3) and (1.4) are used to describe the distribution of the nutrient concentration and the inhibitor concentration, respectively. Accordingly, the problem (1.1) -(1.11) is called the quasi-stationary model. Equation (1.5) is due to the mass conservation law, and the Stokes equation (1.6) subject to the boundary condition (1.7) reflects the motion of tumor cells for the fluid-like tissue. Equation (1.8) is the classical Stefan condition for free boundary $\partial \Omega(t)$, and equations (1.9) and (1.10) indicate six scalar constraints for the system (1.5)-(1.7), since it has six-dimensional kernel of rigid motions $\{\mathbf{a}+\mathbf{b} \times x\}$. The last equation is the initial condition. If the 
stationary diffusion equations (1.1) and (1.2) are replaced by their non-stationary version

$$
\begin{array}{cc}
c_{1} \partial_{t} \sigma=\Delta \sigma-\lambda_{1} \sigma-\beta & \text { in } \Omega(t), t>0, \\
c_{2} \partial_{t} \beta=\Delta \beta-\lambda_{2} \beta & \text { in } \Omega(t), t>0,
\end{array}
$$

where the positive constant $c_{1}$ (resp. $c_{2}$ ) denotes the ratio of the nutrient diffusion time (resp. inhibitor diffusion time) and the tumor-cell doubling time and is very small (cf. [12, 16], 17]), the resulting problem of (1.1)-(1.11) is called the fully non-stationary model.

In the inhibitor-free case (i.e. $\beta=0$ ), it is not difficult to verify that if $0<\hat{\sigma}<\bar{\sigma}$, then the free problem (1.1)-(1.11) has a unique radial stationary solution, which is denoted by $\left(\sigma_{*}, \mathbf{v}_{*}, p_{*}, \Omega_{*}\right)$. For the fully non-stationary model, Friedman established the local well-posedness in Hölder spaces in [16] and proved with his coauthor $\mathrm{Hu}$ in [18] that there exist symmetry-breaking bifurcation branches of solutions bifurcating from the unique radial stationary solution $\left(\sigma_{*}, \mathbf{v}_{*}, p_{*}, \Omega_{*}\right)$. They also proved in [19 that $\left(\sigma_{*}, \mathbf{v}_{*}, p_{*}, \Omega_{*}\right)$ is linearly asymptotically stable for small $\mu / \gamma$, that is, there exists a threshold value $(\mu / \gamma)^{*}$ such that if $\mu / \gamma<(\mu / \gamma)^{*}$, then the trivial solution of the linearization at $\left(\sigma_{*}, \mathbf{v}_{*}, p_{*}, \Omega_{*}\right)$ of the original problem is asymptotically stable, while if $\mu / \gamma>(\mu / \gamma)^{*}$, then the trivial solution is unstable. Later on, $\mathrm{Wu}$ and Cui improved this linear asymptotic stability to asymptotic stability for the quasi-stationary model in [28, and finally extended this asymptotic stability result to the fully non-stationary model for small $c_{1}$ in [29]. The literature 32 is also mentioned for the study of analyticity of the free boundary in time and space variables for the quasi-stationary model.

In this paper we study the inhibitor-present case (i.e. $\beta \neq 0$ ) of the quasistationary model (1.1)-11.11). We expect to determine how inhibitors affect the growth of a tumor with fluid-like tissue. We would like to mention that the study of the effect of inhibitors on tumor growth is a significant topic due to its evident applications to tumor medicine. Indeed, as was pointed out by Byrne and Chaplain in [4], 5], analysis of mathematical models can help medical doctors and researchers to assess the relative merits of different courses of drug treatment and/or chemotherapy. One could see in the forthcoming sections that the inhibitor-present case is more complicated in rigorous analysis than the inhibitor-free case. For previous works concerning the presence of inhibitors, we refer the readers to the literatures [7, [27], where solid tumors with porous medium tissue were considered. By applying a functional analytic method, a delicate spectrum analysis and Cui's local phase theorem for abstract parabolic differential equations with invariance in Banach spaces developed in [9] and [30], we prove that if a radial stationary solution (existence and distribution of radial stationary solutions of (1.1)-(1.11) have been well figured out; see below) is asymptotically stable under small radial perturbations, then there exists a non-negative threshold value $\gamma_{*}$ such that if $\gamma>\gamma_{*}$, then it is still asymptotically stable under small non-radial perturbations, whereas if $0<\gamma<\gamma_{*}$, then it is unstable. Moreover, for the unstable case, there exists a center-stable manifold such that if the transient solution exists globally and is contained in a sufficiently small neighborhood of the radial stationary solution, then it converges exponentially to the radial stationary solution (modulo translation) and its translation lies on this center-stable manifold. It is worth noting that our results indicate an interesting phenomenon that an increasing inhibitor supply has 
a positive effect on the tumor's treatment and can promote the tumor's stability; cf. Remark 1.3 below. It is also pointed out that compared to a solid tumor with porous medium tissue under the action of inhibitors [27], a tumor with fluid-like tissue under the action of inhibitors has its own characteristic; see Remark 1.4 for more details.

To give a precise statement of our main result, let us make some preparations. By scaling we assume that $\mu=1$. We also assume that $\lambda_{1} \neq \lambda_{2}$ for simplicity, since the case $\lambda_{1}=\lambda_{2}$ can be treated similarly. It is not difficult to verify that the radial stationary solution of the problem (1.1)-(1.11), which we denote by $\left(\sigma_{s}, \beta_{s}, \mathbf{v}_{s}, p_{s}, \Omega_{s}\right)$, has the form (cf. [28])

$$
\begin{aligned}
& \sigma_{s}=\sigma_{s}(r)= A_{1} \frac{R_{s} \sinh \left(\sqrt{\lambda}_{1} r\right)}{r \sinh \left(\sqrt{\lambda}_{1} R_{s}\right)}+\left(A_{2}+\nu \bar{\beta}\right) \frac{R_{s} \sinh \left(\sqrt{\lambda}_{2} r\right)}{r \sinh \left(\sqrt{\lambda}_{2} R_{s}\right)}, \\
& \beta_{s}=\beta_{s}(r)= \frac{\bar{\beta} R_{s} \sinh \left(\sqrt{\lambda}_{2} r\right)}{r \sinh \left(\sqrt{\lambda}_{2} R_{s}\right)}, \\
& \mathbf{v}_{s}=\mathbf{v}_{s}(r)= {\left[A_{1} g\left(\sqrt{\lambda}_{1} r\right) \frac{R_{s} \sinh \left(\sqrt{\lambda}_{1} r\right)}{\sinh \left(\sqrt{\lambda}_{1} R_{s}\right)}\right.} \\
&\left.+A_{2} g\left(\sqrt{\lambda}_{2} r\right) \frac{R_{s} \sinh \left(\sqrt{\lambda}_{2} r\right)}{\sinh \left(\sqrt{\lambda}_{2} R_{s}\right)}-\frac{1}{3} \hat{\sigma} r\right] \mathbf{e}_{r}, \\
& p_{s}=p_{s}(r)=\frac{\gamma}{R_{s}}+ \frac{4}{3} \mu\left(A_{1} \frac{R_{s} \sinh \left(\sqrt{\lambda}_{1} r\right)}{r \sinh \left(\sqrt{\lambda}_{1} R_{s}\right)}+A_{2} \frac{R_{s} \sinh \left(\sqrt{\lambda}_{2} r\right)}{r \sinh \left({\sqrt{\lambda_{2}}}_{R_{s}}\right)}-\hat{\sigma}\right), \\
& \Omega_{s}=\left\{x \in \mathbb{R}^{3}:|x|<R_{s}\right\},
\end{aligned}
$$

where $A_{1}=\bar{\sigma}-\left[\bar{\beta} /\left(\lambda_{2}-\lambda_{1}\right)\right], A_{2}=\left[\bar{\beta} /\left(\lambda_{2}-\lambda_{1}\right)\right]-\nu \bar{\beta}, \mathbf{e}_{r}=x / r, g(r)=$ $(r \operatorname{coth} r-1) / r^{2}$, and $R_{s}$ is the root of the equation

$$
f(R):=A_{1} g\left(\sqrt{\lambda}_{1} R\right)+A_{2} g\left(\sqrt{\lambda_{2}} R\right)=\frac{1}{3} \hat{\sigma} .
$$

As was analyzed in [7, equation (1.18) has none, or a unique, or exactly two solutions in certain situations depending on the model coefficients. Substituting each solution $R_{s}$ of (1.18) into the expressions (1.14)-(1.17), one finds that the radial stationary solution $\left(\sigma_{s}, \beta_{s}, \mathbf{v}_{s}, p_{s}, \Omega_{s}\right)$ of the problem (1.1)-11.11) is correspondingly determined. Moreover, for each existent solution $R_{s}>0$ of (1.18), if $f^{\prime}\left(R_{s}\right)<0$, then the corresponding radial stationary solution $\left(\sigma_{s}, \beta_{s}, \mathbf{v}_{s}, p_{s}, \Omega_{s}\right)$ is asymptotically stable under small radial perturbations, while if $f^{\prime}\left(R_{s}\right)>0$, then it is unstable; cf. 7 for more details.

Denote by $W^{m, q}$ and $B_{q q}^{s}$ the usual Sobolev space and Besov space, respectively, where $m \in \mathbb{N}, s \in \mathbb{R}$ and $q>1$. Let $\mathbb{S}^{2}$ denote the unit sphere centered at the origin. Then $\partial \Omega_{s}=R_{s} \mathbb{S}^{2}$. Given $\rho \in C^{1}\left(\mathbb{S}^{2}\right)$ with $\|\rho\|_{C^{1}\left(\mathbb{S}^{2}\right)}$ sufficiently small, we denote

$$
\Omega_{\rho}:=\left\{x \in \mathbb{R}^{3}: r<R_{s}+\rho(\omega), r=|x|, \omega=\frac{x}{|x|} \in \mathbb{S}^{2}\right\} .
$$

For $t \geq 0$ and $\omega \in \mathbb{S}^{2}$, we identify the functions $\rho(t)(\omega)$ with the functions $\rho(t, \omega)$. Then we can define $\Omega(t):=\Omega_{\rho(t)}, \Omega_{0}:=\Omega_{\rho_{0}}$, and rewrite the initial condition (1.11) as

$$
\rho(0, \omega)=\rho_{0}(\omega) \quad \text { for } \quad \omega \in \mathbb{S}^{2} .
$$


Consequently, we can rewrite a transient solution $(\sigma, \beta, \mathbf{v}, p, \Omega)$ and a radial stationary solution $\left(\sigma_{s}, \beta_{s}, \mathbf{v}_{s}, p_{s}, \Omega_{s}\right)$ as $(\sigma, \beta, \mathbf{v}, p, \rho)$ and $\left(\sigma_{s}, \beta_{s}, \mathbf{v}_{s}, p_{s}, 0\right)$, respectively. Observe that the problem (1.1)-(1.11) is invariant under coordinate translations. For the radial stationary solution $\left(\sigma_{s}, \beta_{s}, \mathbf{v}_{s}, p_{s}, 0\right)$ and $x_{0} \in \mathbb{R}^{3}$, we denote by $\left(\sigma_{s\left[x_{0}\right]}, \beta_{s\left[x_{0}\right]}, \mathbf{v}_{s\left[x_{0}\right]}, p_{s\left[x_{0}\right]}, 0_{\left[x_{0}\right]}\right)$ the radial stationary solution induced by the coordinate translation $\left[x \mapsto x+x_{0}\right]$. Similarly, for the transient solution $(\sigma, \beta, \mathbf{v}, p, \rho)$ and $x_{0} \in \mathbb{R}^{3}$, the translated solution is denoted by $\left(\sigma_{\left[x_{0}\right]}, \beta_{\left[x_{0}\right]}, \mathbf{v}_{\left[x_{0}\right]}, p_{\left[x_{0}\right]}, \rho_{\left[x_{0}\right]}\right)$. Finally, we introduce a convergent sequence $\left\{\gamma_{n}\right\}_{n \geq 2}$ as

$$
\begin{aligned}
\gamma_{n}= & \frac{4(n+1)(2 n+3) R_{s}}{n(n+2)(2 n+1)}\left[\bar{\sigma}-\hat{\sigma}-\nu \bar{\beta}-A_{1} \frac{I_{\frac{3}{2}}\left(\sqrt{\lambda}_{1} R_{s}\right) I_{n+\frac{3}{2}}\left(\sqrt{\lambda}_{1} R_{s}\right)}{I_{\frac{1}{2}}\left(\sqrt{\lambda_{1}} R_{s}\right) I_{n+\frac{1}{2}}\left(\sqrt{\lambda}_{1} R_{s}\right)}\right. \\
& \left.-A_{2} \frac{I_{\frac{3}{2}}\left(\sqrt{\lambda}_{2} R_{s}\right) I_{n+\frac{3}{2}}\left(\sqrt{\lambda_{2}} R_{s}\right)}{I_{\frac{1}{2}}\left(\sqrt{\lambda}_{2} R_{s}\right) I_{n+\frac{1}{2}}\left(\sqrt{\lambda}_{2} R_{s}\right)}\right],
\end{aligned}
$$

and define $\gamma_{*}:=\sup \left\{0, \gamma_{2}, \gamma_{3}, \cdots\right\}$, where $I_{m}(r)(m \geq 0)$ represents the modified Bessel function.

The main result of this paper is stated as follows:

Theorem 1.1. Let $\left(\sigma_{s}, \beta_{s}, \mathbf{v}_{s}, p_{s}, 0\right)$ be the radial stationary solution of the problem (1.1) -(1.11). Given $3<q<+\infty$, there hold the following assertions:

(i) If $f^{\prime}\left(R_{s}\right)<0$, then for any positive $\gamma>\gamma_{*}$ the radial stationary solution $\left(\sigma_{s}, \beta_{s}, \mathbf{v}_{s}, p_{s}, 0\right)$ is asymptotically stable in the following sense: There exists a constant $\varepsilon>0$ such that for any $\rho_{0} \in B_{q q}^{4-1 / q}\left(\mathbb{S}^{2}\right)$ satisfying $\left\|\rho_{0}\right\|_{B_{q q}^{4-1 / q}\left(\mathbb{S}^{2}\right)}<\varepsilon$, the problem (1.1)-(1.11) has a unique solution $(\sigma, \beta, \mathbf{v}, p, \rho)$ for all $t \geq 0$, and there are positive constants $\omega, K$ independent of the initial data and a point $x_{0} \in \mathbb{R}^{3}$ uniquely determined by the initial data, such that for all $t \geq 0$,

$$
\begin{aligned}
& \left\|\sigma(\cdot, t)-\sigma_{s\left[x_{0}\right]}\right\|_{W^{2, q}(\Omega(t))}+\left\|\beta(\cdot, t)-\beta_{s\left[x_{0}\right]}\right\|_{W^{2, q}(\Omega(t))} \\
& +\left\|\mathbf{v}(\cdot, t)-\mathbf{v}_{s\left[x_{0}\right]}\right\|_{W^{3, q}(\Omega(t))}+\left\|p(\cdot, t)-p_{s\left[x_{0}\right]}\right\|_{W^{2, q}(\Omega(t))} \\
& +\left\|\rho(\cdot, t)-0_{\left[x_{0}\right]}\right\|_{B_{q q}^{4-1 / q}\left(\mathbb{S}^{2}\right)} \leq K \mathrm{e}^{-\omega t} .
\end{aligned}
$$

(ii) If $f^{\prime}\left(R_{s}\right)>0$ or $0<\gamma<\gamma_{*}$, then the radial stationary solution $\left(\sigma_{s}, \beta_{s}, \mathbf{v}_{s}\right.$, $\left.p_{s}, 0\right)$ is unstable. Moreover, if further $\gamma \notin\left\{\gamma_{n}\right\}_{n \geq 2}$, then there exist two small neighborhoods $O_{1}$ and $O_{2}, O_{2} \subseteq O_{1}$, of 0 in $B_{q q}^{4-1 / q}\left(\mathbb{S}^{2}\right)$ and a center-stable manifold $\mathcal{M}$ contained in $O_{1}$ such that for any $\rho_{0} \in O_{2}$, if the transient solution $(\sigma, \beta, \mathbf{v}, p, \rho)$ exists globally and $\rho(t) \in O_{1}$ for all $t \geq 0$, then there exist two constants $\omega, K$ independent of $\rho_{0}$ and a point $x_{0} \in \mathbb{R}^{3}$ uniquely determined by $\rho_{0}$ such that (1.21) holds and $\rho_{\left[x_{0}\right]}(t) \in \mathcal{M}$, for all $t \geq 0$.

Remark 1.2. Based on the results obtained in Section 4, by some suitable modifications (with no essential difficulties but very complicated calculations) of the framework of [29], we can extend the results of this paper to the fully non-stationary model for small $c_{1}$ and $c_{2}$.

Remark 1.3. It is worth noting that the threshold value $\gamma_{*}$ is a monotone decreasing function of the inhibitor supple $\bar{\beta}$ (cf. Section 4), which means that an increasing inhibitor supply has a positive effect on the tumor's treatment and it can promote the tumor's stability.

Remark 1.4. It is interesting to compare the threshold value $\gamma_{*}$ with the corresponding one, which we denote by $\tilde{\gamma}_{*}$, obtained in [27] for a solid tumor model 
with porous medium tissue. We find that a tumor with fluid-like tissue has its own characteristics. In fact, similar stability results have been obtained in [27]: If a radial stationary solution is asymptotically stable under radial perturbations, then there exists a threshold value $\tilde{\gamma}_{*}$ such that if $\gamma>\tilde{\gamma}_{*}$, then it remains asymptotically stable under non-radial perturbations, whereas if $0<\gamma<\tilde{\gamma}_{*}$, then it is unstable. We show that if $0<R_{s}<\sqrt{210} / 5$, then $\gamma_{*}>\tilde{\gamma}_{*}$, while $\gamma_{*}<\tilde{\gamma}_{*}$ if $R_{s}$ is large enough (see Lemma 4.8 for more details). From the point of view of modeling, this implies that a radial solid tumor with porous medium tissue in the dormant state is more stable than a radial tumor with fluid-like tissue for small $R_{s}$, whereas for large enough $R_{s}$ this is reversed.

The structure of the rest of this paper is arranged as follows. In the next section, we transform the free boundary problem (1.1)-(1.11) into an initial-boundary value problem defined on a fixed reference domain. In Section 3 we further reduce the problem obtained in Section 2 into a Cauchy problem of an abstract differential equation in Banach space and establish its local well-posedness. Section 4 aims at investigating the linearization of (1.1)-(1.11) at the radial stationary solution and calculating the spectrum of the reduced linearized operator. In the last section we study the asymptotic behavior of solutions of (1.1)-(1.11) and give the proof of the main result.

\section{TRANSFORMATION}

In this section we convert the free boundary problem (1.1)-11.11) into an initialboundary value problem on the fixed reference domain $\Omega_{s}$.

Let $3<q<+\infty$ be given as in Theorem 1.1. Let $a_{0} \in\left(0, R_{s} / 4\right)$ and

$$
\mathcal{O}:=\left\{\rho \in B_{q q}^{4-1 / q}\left(\mathbb{S}^{2}\right),\|\rho\|_{C^{1}\left(\mathbb{S}^{2}\right)}<a_{0}\right\} .
$$

By Sobolev embedding theorem we have $B_{q q}^{4-1 / q}\left(\mathbb{S}^{2}\right) \subset C^{3}\left(\mathbb{S}^{2}\right)$, and $B_{q q}^{4-1 / q}\left(\mathbb{S}^{2}\right)$ is a Banach algebra. For each given $\rho \in \mathcal{O}$, we define a mapping

$$
\psi_{\rho}: \partial \Omega_{s} \rightarrow \mathbb{R}^{3}, \quad x \mapsto x+\rho(\omega) \omega,
$$

where $\omega=x /|x|$. Recall that $\Omega_{\rho}=\left\{x \in \mathbb{R}^{3}:|x|<R_{s}+\rho(\omega), \omega \in \mathbb{S}^{2}\right\}$ and $\partial \Omega_{s}=R_{s} \mathbb{S}^{2}$. It is easy to see that $\operatorname{Im}\left(\psi_{\rho}\right)=\partial \Omega_{\rho}$. Moreover, $\psi_{\rho}$ is near the identity and $\psi_{\rho} \in \operatorname{Diff}^{3}\left(\partial \Omega_{s}, \partial \Omega_{\rho}\right)$, provided $a_{0}>0$ is sufficiently small. Since we only consider the small non-radial perturbation of radial stationary solution, we assume there exists $\rho_{0} \in \mathcal{O}$ such that $\partial \Omega_{0}=\operatorname{Im}\left(\psi_{\rho_{0}}\right)$.

Let $\pi^{*}: C\left(\partial \Omega_{s}\right) \rightarrow C\left(\mathbb{S}^{2}\right)$ and $\pi_{*}: C\left(\mathbb{S}^{2}\right) \rightarrow C\left(\partial \Omega_{s}\right)$ be the pull-back and pushforward operators induced by the natural projection from $\mathbb{S}^{2}$ to $\partial \Omega_{s}$, respectively, i.e.,

$$
\begin{array}{ll}
\left(\pi^{*} \eta\right)(\omega):=\eta\left(R_{s} \omega\right) & \text { for } \eta \in C\left(\partial \Omega_{s}\right), \\
\left(\pi_{*} \rho\right)\left(R_{s} \omega\right):=\rho(\omega) & \text { for } \rho \in C\left(\mathbb{S}^{2}\right) .
\end{array}
$$

Given $\hat{\rho} \in B_{q q}^{4-1 / q}\left(\partial \Omega_{s}\right)$, we define a mapping $\Pi(\hat{\rho}):=u$, where $u$ is the solution of the following elliptic boundary value problem:

$$
\Delta u=0 \text { in } \Omega_{s}, \quad u=\hat{\rho} \text { on } \partial \Omega_{s} .
$$

By the well-known theory of elliptic differential equations, we see $\Pi \in L\left(B_{q q}^{4-1 / q}\left(\partial \Omega_{s}\right)\right.$, $W^{4, q}\left(\Omega_{s}\right)$ ). For each given $\rho \in \mathcal{O}$, we define a mapping $\Psi_{\rho}: \Omega_{s} \rightarrow \Omega_{\rho}$ by

$$
\Psi_{\rho}:=\operatorname{Id}_{\Omega_{s}}+\Pi\left(\psi_{\rho}-\operatorname{Id}_{\partial \Omega_{s}}\right) .
$$


It is clear that $\left.\Psi_{\rho}\right|_{\partial \Omega_{s}}=\psi_{\rho}$ and $\Psi_{\rho} \in \operatorname{Diff}^{4, q}\left(\Omega_{s}, \Omega_{\rho}\right)$. Moreover, $\Psi_{\rho}$ is near the identity and

$$
\left[\rho \mapsto \Psi_{\rho}\right] \in C^{\infty}\left(\mathcal{O},\left(W^{4, q}\left(\Omega_{\rho}\right)\right)^{3}\right),
$$

provided that $a_{0}$ is sufficiently small. We denote by $\Psi_{\rho}^{*}$ and $\Psi_{*}^{\rho}$ the pull-back and push-forward operators induced by $\Psi_{\rho}$, respectively, i.e.,

$$
\begin{array}{ll}
\Psi_{\rho}^{*} u:=u \circ \Psi_{\rho} & \text { for } \quad u \in C\left(\Omega_{\rho}\right), \\
\Psi_{*}^{\rho} v:=v \circ \Psi_{\rho}^{-1} & \text { for } \quad v \in C\left(\Omega_{s}\right) .
\end{array}
$$

Let $\rho \in \mathcal{O}$ be given. We introduce the following transformed differential operators as in [28]:

$$
\begin{array}{ll}
\mathcal{A}(\rho) u=\Psi_{\rho}^{*}\left(\Delta\left(\Psi_{*}^{\rho} u\right)\right), & \overrightarrow{\mathcal{B}}(\rho) u=\Psi_{\rho}^{*}\left(\nabla\left(\Psi_{*}^{\rho} u\right)\right), \\
\overrightarrow{\mathcal{B}}(\rho) \cdot \mathbf{v}=\Psi_{\rho}^{*}\left(\nabla \cdot\left(\Psi_{*}^{\rho} \mathbf{v}\right)\right), & \overrightarrow{\mathcal{B}}(\rho) \otimes \mathbf{v}=\Psi_{\rho}^{*}\left(\nabla \otimes\left(\Psi_{*}^{\rho} \mathbf{v}\right)\right),
\end{array}
$$

for scalar functions $u \in W^{2, q}\left(\Omega_{s}\right)$ and vector functions $\mathbf{v}=\left(v_{1}, v_{2}, v_{3}\right) \in\left(W^{1, q}\left(\Omega_{s}\right)\right)^{3}$. More precisely, by letting

$$
a_{i j}^{\rho}:=\left[D \Psi_{\rho}\right]_{i j}^{-1}, \quad i, j=1,2,3, \quad \text { and } \quad G_{\rho}:=\operatorname{det}\left(D \Psi_{\rho}\right),
$$

the operators $\mathcal{A}(\rho), \overrightarrow{\mathcal{B}}(\rho), \overrightarrow{\mathcal{B}}(\rho) \cdot$ and $\overrightarrow{\mathcal{B}}(\rho) \otimes$ have the expressions as

$$
\left\{\begin{array}{l}
\mathcal{A}(\rho) u=\sum_{i, j, k=1}^{3} a_{i j}^{\rho} \partial_{j}\left(a_{i k}^{\rho} \partial_{k} u\right), \\
\overrightarrow{\mathcal{B}}(\rho) u=\left(\sum_{j=1}^{3} a_{1 j}^{\rho} \partial_{j} u, \sum_{j=1}^{3} a_{2 j}^{\rho} \partial_{j} u, \sum_{j=1}^{3} a_{3 j}^{\rho} \partial_{j} u\right), \\
\overrightarrow{\mathcal{B}}(\rho) \cdot \mathbf{v}=\sum_{i, j=1}^{3} a_{i j}^{\rho} \partial_{j} v_{i}, \quad \overrightarrow{\mathcal{B}}(\rho) \otimes \mathbf{v}=\left(\sum_{k=1}^{3} a_{i k}^{\rho} \partial_{k} v_{j}\right)_{3 \times 3}
\end{array} .\right.
$$

Clearly, for sufficiently small $a_{0}>0$,

$$
\left\{\begin{array}{l}
{\left[\rho \mapsto a_{i j}^{\rho}\right] \in C^{\infty}\left(\mathcal{O}, W^{3, q}\left(\Omega_{s}\right)\right), i, j=1,2,3,} \\
{\left[\rho \mapsto G_{\rho}\right] \in C^{\infty}\left(\mathcal{O}, W^{3, q}\left(\Omega_{s}\right)\right) .}
\end{array}\right.
$$

Then by the Sobolev embedding theorem and the properties of the Banach algebra $W^{m, q}\left(\Omega_{s}\right)$ for $m \geq 1$ and $q>3$, we can easily verify that

$$
\left\{\begin{array}{l}
{[\rho \mapsto \mathcal{A}(\rho)] \in C^{\infty}\left(\mathcal{O}, L\left(W^{2, q}\left(\Omega_{s}\right), L^{q}\left(\Omega_{s}\right)\right)\right)} \\
{[\rho \mapsto \overrightarrow{\mathcal{B}}(\rho)] \in C^{\infty}\left(\mathcal{O}, L\left(W^{2, q}\left(\Omega_{s}\right),\left(W^{1, q}\left(\Omega_{s}\right)\right)^{3}\right)\right),} \\
{[\rho \mapsto \overrightarrow{\mathcal{B}}(\rho) \cdot] \in C^{\infty}\left(\mathcal{O}, L\left(\left(W^{2, q}\left(\Omega_{s}\right)\right)^{3}, W^{1, q}\left(\Omega_{s}\right)\right)\right)} \\
{[\rho \mapsto \overrightarrow{\mathcal{B}}(\rho) \otimes] \in C^{\infty}\left(\mathcal{O}, L\left(\left(W^{2, q}\left(\Omega_{s}\right)\right)^{3},\left(W^{1, q}\left(\Omega_{s}\right)\right)^{3 \times 3}\right)\right) .}
\end{array}\right.
$$

Next, for the unit outward normal $\mathbf{n}$ and the mean curvature $\kappa$ of $\partial \Omega_{\rho}$, we define

$$
\widetilde{\mathbf{n}}_{\rho}(x):=\mathbf{n}\left(\psi_{\rho}(x)\right) \text { and } \quad \widetilde{\kappa}_{\rho}(x):=\kappa\left(\psi_{\rho}(x)\right), \quad \text { for } x \in \partial \Omega_{s} .
$$


A simple calculation shows that

$$
\left\{\begin{array}{l}
\widetilde{\mathbf{n}}_{\rho}(x) \equiv\left(\widetilde{\mathbf{n}}_{\rho}^{1}(x), \widetilde{\mathbf{n}}_{\rho}^{2}(x), \widetilde{\mathbf{n}}_{\rho}^{3}(x)\right)=\frac{\sum_{i, j=1}^{3} a_{i j}^{\rho}(x) x_{j} \mathbf{e}_{i}}{\left|\sum_{i, j=1}^{3} a_{i j}^{\rho}(x) x_{j} \mathbf{e}_{i}\right|} \\
\widetilde{\kappa}_{\rho}(x)=\frac{1}{2} \sum_{i, j=1}^{3} a_{i j}^{\rho}(x) \partial_{j} \widetilde{\mathbf{n}}_{\rho}^{i}(x),
\end{array}\right.
$$

where $\mathbf{e}_{1}=(1,0,0), \mathbf{e}_{2}=(0,1,0)$ and $\mathbf{e}_{3}=(0,0,1)$. By (2.3) and (2.5) we have

$$
\left\{\begin{array}{l}
{\left[\rho \mapsto \widetilde{\mathbf{n}}_{\rho}\right] \in C^{\infty}\left(\mathcal{O},\left(B_{q q}^{3-1 / q}\left(\partial \Omega_{s}\right)\right)^{3}\right),} \\
{\left[\rho \mapsto \widetilde{\kappa}_{\rho}\right] \in C^{\infty}\left(\mathcal{O}, B_{q q}^{2-1 / q}\left(\partial \Omega_{s}\right)\right) .}
\end{array}\right.
$$

Let $T>0$ be given and consider a function $\rho \in C([0, T], \mathcal{O})$. Set

$$
\Omega(t):=\Omega_{\rho(t)} \quad \text { and } \quad \partial \Omega(t):=\partial \Omega_{\rho(t)}, \quad \text { for } \quad t \in[0, T] .
$$

Then

$$
\partial \Omega(t)=\operatorname{Im} \psi_{\rho(t)}=\left\{x \in \mathbb{R}^{3}: x=r \omega, r=R_{s}+\rho(t, \omega), \omega \in \mathbb{S}^{2}\right\}
$$

where $r=|x|$ and $\omega=x /|x|$. Define a function $\phi_{\rho}:[0, T] \times \mathbb{R}^{3} \backslash\{0\} \rightarrow \mathbb{R}$ by

$$
\phi_{\rho}(t, x):=r-R_{s}-\rho(t, \omega)
$$

We have $x \in \partial \Omega(t)$ if and only if $\phi_{\rho}(t, x)=0$. It follows that for $t \in[0, T]$ and $x \in \partial \Omega(t)$, the unit outward normal field $\mathbf{n}$ and the normal velocity $V_{\mathbf{n}}$ of $\partial \Omega(t)$ are given by

$$
\mathbf{n}(t, x)=\frac{\nabla_{x} \phi_{\rho}(t, x)}{\left|\nabla_{x} \phi_{\rho}(t, x)\right|} \quad \text { and } \quad V_{\mathbf{n}}(t, x)=\frac{\partial_{t} \rho(t, \omega)}{\left|\nabla_{x} \phi_{\rho}(t, x)\right|}
$$

Substituting the above formulas into (1.8), we obtain

$$
\partial_{t} \rho(t, \omega)=\mathbf{v}(t, x) \cdot \nabla_{x} \phi_{\rho}(t, x), \quad \text { for } \quad t \in[0, T], x \in \partial \Omega(t),
$$

where $\omega=x /|x|$. Set $\mathbf{w}_{1}(x):=\left(0, x_{3},-x_{2}\right), \mathbf{w}_{2}(x):=\left(-x_{3}, 0, x_{1}\right)$ and $\mathbf{w}_{3}(x):=$ $\left(x_{2},-x_{1}, 0\right)$. Note that $\mathbf{v} \times x=\left(\mathbf{v} \cdot \mathbf{w}_{1}, \mathbf{v} \cdot \mathbf{w}_{2}, \mathbf{v} \cdot \mathbf{w}_{3}\right)$. We denote $\widetilde{\mathbf{w}}_{j}^{\rho}:=\Psi_{\rho}^{*} \mathbf{w}_{j}$, $j=1,2,3$. Finally, for $\sigma, \beta, \mathbf{v}$ and $p$ as in problem (1.1) (1.11), we denote

$$
\widetilde{\sigma}:=\Psi_{\rho}^{*} \sigma, \quad \widetilde{\beta}:=\Psi_{\rho}^{*} \beta, \quad \widetilde{\mathbf{v}}:=\Psi_{\rho}^{*} \mathbf{v}, \quad \widetilde{p}:=\Psi_{\rho}^{*} p,
$$

and $\widetilde{\mathbf{T}}_{\rho}(\widetilde{\mathbf{v}}, \widetilde{p}):=\left[\overrightarrow{\mathcal{B}}(\rho) \otimes \widetilde{\mathbf{v}}+(\overrightarrow{\mathcal{B}}(\rho) \otimes \widetilde{\mathbf{v}})^{T}\right]-[\widetilde{p}+(2 / 3) \overrightarrow{\mathcal{B}}(\rho) \cdot \widetilde{\mathbf{v}}] \mathbf{I}$. 
Using this notation, we convert the free boundary problem (1.1)-(1.11) into the following problem on the fixed domain $\Omega_{s} \times[0, T]$ :

$$
\begin{aligned}
& \mathcal{A}(\rho) \tilde{\sigma}=\lambda_{1} \widetilde{\sigma}+\widetilde{\beta} \quad \text { in } \Omega_{s} \times[0, T], \\
& \mathcal{A}(\rho) \widetilde{\beta}=\lambda_{2} \widetilde{\beta} \quad \text { in } \Omega_{s} \times[0, T], \\
& \widetilde{\sigma}=\bar{\sigma} \quad \text { on } \partial \Omega_{s} \times[0, T] \text {, } \\
& \widetilde{\beta}=\bar{\beta} \quad \text { on } \partial \Omega_{s} \times[0, T], \\
& \overrightarrow{\mathcal{B}}(\rho) \cdot \widetilde{\mathbf{v}}=(\widetilde{\sigma}-\hat{\sigma})-\nu \widetilde{\beta} \quad \text { in } \Omega_{s} \times[0, T], \\
& -\mathcal{A}(\rho) \widetilde{\mathbf{v}}+\overrightarrow{\mathcal{B}}(\rho) \widetilde{p}-\frac{1}{3} \overrightarrow{\mathcal{B}}(\rho)(\overrightarrow{\mathcal{B}}(\rho) \cdot \widetilde{\mathbf{v}})=0 \quad \text { in } \Omega_{s} \times[0, T], \\
& \widetilde{\mathbf{T}}_{\rho}(\widetilde{\mathbf{v}}, \widetilde{p}) \widetilde{\mathbf{n}}_{\rho}=-\gamma \widetilde{\kappa}_{\rho} \widetilde{\mathbf{n}}_{\rho} \quad \text { on } \partial \Omega_{s} \times[0, T], \\
& \int_{\Omega_{s}} \widetilde{\mathbf{v}}(t, x) G_{\rho}(t, x) \mathrm{d} x=0, \quad \text { for } t \in[0, T] \text {, } \\
& \int_{\Omega_{s}} \widetilde{\mathbf{v}}(t, x) \cdot \widetilde{\mathbf{w}}_{j}^{\rho}(t, x) G_{\rho}(t, x) \mathrm{d} x=0, \quad j=1,2,3, \quad \text { for } t \in[0, T], \\
& \partial_{t} \rho(t, \omega)=\pi^{*}\left(\widetilde{\mathbf{v}} \cdot\left[\omega-\frac{R_{s}}{R_{s}+\rho} \nabla_{\omega} \rho\right]\right) \quad \text { on } \partial \Omega_{s} \times[0, T], \\
& \rho(0)=\rho_{0} \text {. }
\end{aligned}
$$

Notice that we have used the formula $\nabla_{x} \phi_{\rho}=\partial_{r} \phi_{\rho} \cdot \omega+r^{-1} \nabla_{\omega} \phi_{\rho}$ for the spherical polar coordinate $(r, \omega)$, the transformation (2.1) and equation (2.7) to get (2.17).

In summary, we have the following elementary result:

Lemma 2.1. Problem (1.1)-(1.11) and problem (2.8)-(2.18) are equivalent in the following sense: If $(\sigma, \beta, \mathbf{v}, p, \rho)$ is a solution of problem (1.1) -(1.11), then by setting

$$
\widetilde{\sigma}=\sigma \circ \Psi_{\rho}, \quad \widetilde{\beta}=\beta \circ \Psi_{\rho}, \quad \widetilde{\mathbf{v}}=\mathbf{v} \circ \Psi_{\rho}, \quad \widetilde{p}=p \circ \Psi_{\rho},
$$

we have that $(\widetilde{\sigma}, \widetilde{\beta}, \widetilde{\mathbf{v}}, \widetilde{p}, \rho)$ is a solution of problem (2.8)-(2.18). Conversely, if $(\widetilde{\sigma}, \widetilde{\beta}, \widetilde{\mathbf{v}}, \widetilde{p}, \rho)$ is a solution of problem (2.8) $-(2.18)$, then by setting

$$
\sigma=\widetilde{\sigma} \circ \Psi_{\rho}^{-1}, \quad \beta=\widetilde{\beta} \circ \Psi_{\rho}^{-1}, \quad \mathbf{v}=\widetilde{\mathbf{v}} \circ \Psi_{\rho}^{-1}, \quad p=\widetilde{p} \circ \Psi_{\rho}^{-1},
$$

we have that $(\sigma, \beta, \mathbf{v}, p, \rho)$ is a solution of problem (1.1)-(1.11).

\section{REDUCTION}

In this section we reduce problem (2.8) -(2.18) into a Cauchy problem of an abstract differential equation in Banach space, which contains the unknown function $\rho$ only. We shall also prove that this equation is of parabolic type and establish its local well-posedness.

We first give a preliminary result:

Lemma 3.1. Let $a_{0}>0$ be sufficiently small. For given $\rho \in \mathcal{O}$, the boundary value problem (2.8)-(2.11) has a unique solution $(\widetilde{\sigma}, \widetilde{\beta})=(\mathcal{R}(\rho), \mathcal{T}(\rho)) \in W^{4, q}\left(\Omega_{s}\right) \times$ $W^{4, q}\left(\Omega_{s}\right)$. Moreover,

$$
\mathcal{R} \in C^{\infty}\left(\mathcal{O}, W^{4, q}\left(\Omega_{s}\right)\right), \quad \mathcal{T} \in C^{\infty}\left(\mathcal{O}, W^{4, q}\left(\Omega_{s}\right)\right) .
$$


Proof. For given $\rho \in \mathcal{O}$, it follows from (2.2) that $\mathcal{A}(\rho)$ is the Laplace-Beltrami operator on $\Omega_{s}$ with respect to the metric induced by $\Psi_{\rho}$. By applying the standard $L^{p}$ theory for elliptic boundary value problems we get that problem (2.9) and (2.11) has a unique solution $\widetilde{\beta}=\mathcal{T}(\rho) \in W^{4, q}\left(\Omega_{s}\right)$. Moreover, with a slight modification of the proof of Theorem 3.1 in [10], we have $\mathcal{T} \in C^{\infty}\left(\mathcal{O}, W^{4, q}\left(\Omega_{s}\right)\right)$. Substituting $\mathcal{T}(\rho)$ into (2.8) and deriving similarly as above, we obtain that problem (2.8) and (2.10) has a unique solution $\tilde{\sigma}=\mathcal{R}(\rho)$ satisfying $\mathcal{R} \in C^{\infty}\left(\mathcal{O}, W^{4, q}\left(\Omega_{s}\right)\right)$. We also refer to Lemma 3.1 in 8 .

Next, we substitute the above obtained $\widetilde{\sigma}=\mathcal{R}(\rho)$ and $\widetilde{\beta}=\mathcal{T}(\rho)$ into (2.12), and solve the Stokes problem (2.12)-(2.16). To this aim, for given $\rho \in \mathcal{O}$, we consider a more general problem:

$$
\begin{cases}\overrightarrow{\mathcal{B}}(\rho) \cdot \widetilde{\mathbf{v}}=\varphi & \text { in } \Omega_{s}, \\ -\mathcal{A}(\rho) \widetilde{\mathbf{v}}+\overrightarrow{\mathcal{B}}(\rho) \widetilde{p}=\mathbf{g} & \text { in } \Omega_{s}, \\ \widetilde{\mathbf{T}}_{\rho}(\widetilde{\mathbf{v}}, \widetilde{p}) \widetilde{\mathbf{n}}_{\rho}=\mathbf{h} & \text { on } \partial \Omega_{s}, \\ \int_{\Omega_{s}} \widetilde{\mathbf{v}}(x) G_{\rho}(x) \mathrm{d} x=0, & \\ \int_{\Omega_{s}} \widetilde{\mathbf{v}}(x) \cdot \widetilde{\mathbf{w}}_{j}^{\rho}(x) G_{\rho}(x) \mathrm{d} x=0, & j=1,2,3,\end{cases}
$$

where $\varphi \in W^{2, q}\left(\Omega_{s}\right), \mathbf{g} \in\left(W^{1, q}\left(\Omega_{s}\right)\right)^{3}$ and $\mathbf{h} \in\left(B_{q q}^{2-1 / q}\left(\partial \Omega_{s}\right)\right)^{3}$. By a slight modification to the proofs of Lemma 2.3 and Lemma 2.4 in [28], we can easily prove that

Lemma 3.2. Let $a_{0}>0$ be sufficiently small. For given $\rho \in \mathcal{O}$, if there holds

$$
\left\{\begin{array}{l}
\int_{\Omega_{s}}\left(\mathbf{g}(x)-\frac{1}{3} \overrightarrow{\mathcal{B}}(\rho) \varphi(x)\right) \cdot \widetilde{\mathbf{w}}_{j}^{\rho}(x) G_{\rho}(x) \mathrm{d} x+\int_{\partial \Omega_{s}} \mathbf{h}(\xi) \cdot \widetilde{\mathbf{w}}_{j}^{\rho}(\xi) H_{\rho}(\xi) \mathrm{d} \xi=0, \\
\int_{\Omega_{s}}\left(\mathbf{g}(x)-\frac{1}{3} \overrightarrow{\mathcal{B}}(\rho) \varphi(x)\right) \cdot \mathbf{e}_{j} G_{\rho}(x) \mathrm{d} x+\int_{\partial \Omega_{s}} \mathbf{h}(\xi) \cdot \mathbf{e}_{j} H_{\rho}(\xi) \mathrm{d} \xi=0, j=1,2,3,
\end{array}\right.
$$

where $H_{\rho}=\left|\psi_{\rho}\right|^{2} \sqrt{1+\left|\nabla_{\omega} \psi_{\rho}\right|^{2}}$ is the Jacobian induced by $\psi_{\rho}$ on $\partial \Omega_{s}$, then problem (3.2) has a unique solution $(\widetilde{\mathbf{v}}, \widetilde{p}) \in\left(W^{3, q}\left(\Omega_{s}\right)\right)^{3} \times W^{2, q}\left(\Omega_{s}\right)$. Moreover, we have

$$
\widetilde{\mathbf{v}}=\mathbf{P}(\rho) \varphi+\mathbf{Q}(\rho) \mathbf{g}+\mathbf{R}(\rho) \mathbf{h}, \quad \widetilde{p}=\mathcal{S}(\rho)(\varphi, \mathbf{g}, \mathbf{h})
$$

and

$$
\left\{\begin{array}{l}
\mathbf{P} \in C^{\infty}\left(\mathcal{O}, L\left(W^{2, q}\left(\Omega_{s}\right),\left(W^{3, q}\left(\Omega_{s}\right)\right)^{3}\right)\right), \\
\mathbf{Q} \in C^{\infty}\left(\mathcal{O}, L\left(\left(W^{1, q}\left(\Omega_{s}\right)\right)^{3},\left(W^{3, q}\left(\Omega_{s}\right)\right)^{3}\right)\right), \\
\mathbf{R} \in C^{\infty}\left(\mathcal{O}, L\left(\left(B_{q q}^{2-1 / q}\left(\partial \Omega_{s}\right)\right)^{3},\left(W^{3, q}\left(\Omega_{s}\right)\right)^{3}\right)\right), \\
\mathcal{S} \in C^{\infty}\left(\mathcal{O}, L\left(W^{2, q}\left(\Omega_{s}\right) \times\left(W^{1, q}\left(\Omega_{s}\right)\right)^{3} \times\left(B_{q q}^{2-1 / q}\left(\partial \Omega_{s}\right)\right)^{3}, W^{2, q}\left(\Omega_{s}\right)\right)\right) .
\end{array}\right.
$$


We rewrite problem (2.12)-(2.16) in the form of (3.2), with

$$
\varphi=\mathcal{R}(\rho)-\hat{\sigma}-\nu \mathcal{T}(\rho), \quad \mathbf{g}=\frac{1}{3} \overrightarrow{\mathcal{B}}(\rho)(\mathcal{R}(\rho)-\hat{\sigma}-\nu \mathcal{T}(\rho)), \quad \mathbf{h}=-\gamma \widetilde{\kappa}_{\rho} \widetilde{\mathbf{n}}_{\rho} .
$$

By Lemma 3.1, we immediately have $\varphi \in W^{2, q}\left(\Omega_{s}\right), \mathbf{g} \in\left(W^{1, q}\left(\Omega_{s}\right)\right)^{3}$ and $\mathbf{h} \in$ $\left(B_{q q}^{2-1 / q}\left(\partial \Omega_{s}\right)\right)^{3}$. By (48) and (49) in [28], we have

$\int_{\partial \Omega_{s}} \widetilde{\kappa}_{\rho}(\xi) \widetilde{\mathbf{n}}_{\rho}(\xi) \cdot \widetilde{\mathbf{w}}_{j}^{\rho}(\xi) H_{\rho}(\xi) \mathrm{d} \xi=0, \quad \int_{\partial \Omega_{s}} \widetilde{\kappa}_{\rho}(\xi) \widetilde{\mathbf{n}}_{\rho}(\xi) \cdot \mathbf{e}_{j} H_{\rho}(\xi) \mathrm{d} \xi=0, \quad j=1,2,3$.

This implies that (3.3) holds for $\varphi, \mathbf{g}$ and $\mathbf{h}$ given by (3.6). Hence it follows from Lemma 3.2 that problem (2.12) $-(2.16)$ has a unique solution $(\widetilde{\mathbf{v}}, \widetilde{p}) \in W^{3, q}\left(\Omega_{s}\right) \times$ $W^{2, q}\left(\Omega_{s}\right)$ in the form

$$
\left\{\begin{aligned}
\widetilde{\mathbf{v}}= & \mathbf{P}(\rho)(\mathcal{R}(\rho)-\hat{\sigma}-\nu \mathcal{T}(\rho))+\frac{1}{3} \mathbf{Q}(\rho) \overrightarrow{\mathcal{B}}(\rho)(\mathcal{R}(\rho)-\hat{\sigma}-\nu \mathcal{T}(\rho)) \\
& -\gamma \mathbf{R}(\rho)(\mathcal{K}(\rho) \overrightarrow{\mathcal{N}}(\rho)) \\
\widetilde{p}= & \mathcal{S}(\rho)\left(\mathcal{R}(\rho)-\hat{\sigma}-\nu \mathcal{T}(\rho), \frac{1}{3} \overrightarrow{\mathcal{B}}(\rho)(\mathcal{R}(\rho)-\hat{\sigma}-\nu \mathcal{T}(\rho)),-\gamma \mathcal{K}(\rho) \overrightarrow{\mathcal{N}}(\rho)\right)
\end{aligned}\right.
$$

where $\mathcal{K}(\rho)=\widetilde{\kappa}_{\rho}$ and $\overrightarrow{\mathcal{N}}(\rho)=\widetilde{\mathbf{n}}_{\rho}$. By (2.6) we see

$$
\mathcal{K} \in C^{\infty}\left(\mathcal{O}, B_{q q}^{2-1 / q}\left(\partial \Omega_{s}\right)\right), \quad \overrightarrow{\mathcal{N}} \in C^{\infty}\left(\mathcal{O},\left(B_{q q}^{3-1 / q}\left(\partial \Omega_{s}\right)\right)^{3}\right) .
$$

Substitute the obtained $\widetilde{\mathbf{v}}$ in (3.7) into (2.17), and define a mapping $\mathcal{Q}: \mathcal{O} \rightarrow$ $B_{q q}^{3-1 / q}\left(\mathbb{S}^{2}\right)$ by

$$
\begin{aligned}
\mathcal{Q}(\rho)= & \pi^{*}\left(\mathbf{P}(\rho)(\mathcal{R}(\rho)-\hat{\sigma}-\nu \mathcal{T}(\rho))+\frac{1}{3} \mathbf{Q}(\rho) \overrightarrow{\mathcal{B}}(\rho)(\mathcal{R}(\rho)-\hat{\sigma}-\nu \mathcal{T}(\rho))\right. \\
& -\gamma \mathbf{R}(\rho)(\mathcal{K}(\rho) \overrightarrow{\mathcal{N}}(\rho)))\left.\right|_{\partial \Omega_{s}} \cdot\left(\omega-\frac{R_{s}}{R_{s}+\rho} \nabla_{\omega} \rho\right), \quad \text { for } \rho \in \mathcal{O} .
\end{aligned}
$$

It follows from (2.4), (3.1), (3.5), (3.7) and (3.8) that

$$
\mathcal{Q} \in C^{\infty}\left(\mathcal{O}, B_{q q}^{3-1 / q}\left(\mathbb{S}^{2}\right)\right)
$$

Thus problem (2.8)-(2.18) is reduced into the following Cauchy problem for a differential equation in the Banach space $B_{q q}^{3-1 / q}\left(\mathbb{S}^{2}\right)$ :

$$
\left\{\begin{array}{l}
\frac{\mathrm{d} \rho}{\mathrm{d} t}=\mathcal{Q}(\rho), \quad t>0, \\
\rho(0)=\rho_{0} .
\end{array}\right.
$$

We summarize:

Lemma 3.3. Problem (2.8)-(2.18) and problem (3.10) are equivalent in the following sense: If $(\widetilde{\sigma}, \widetilde{\beta}, \widetilde{\mathbf{v}}, \widetilde{p}, \rho)$ is a solution of problem (2.8) -(2.18), then $\rho$ is a solution of problem (3.10). Conversely, if $\rho$ is a solution of problem (3.10), then by setting

$$
\left\{\begin{array}{l}
\widetilde{\sigma}=\mathcal{R}(\rho), \quad \widetilde{\beta}=\mathcal{T}(\rho) \\
\widetilde{\mathbf{v}}=\mathbf{P}(\rho)(\widetilde{\sigma}-\hat{\sigma}-\nu \widetilde{\beta})+\frac{1}{3} \mathbf{Q}(\rho) \overrightarrow{\mathcal{B}}(\rho)(\widetilde{\sigma}-\hat{\sigma}-\nu \widetilde{\beta})-\gamma \mathbf{R}(\rho)(\mathcal{K}(\rho) \overrightarrow{\mathcal{N}}(\rho)), \\
\widetilde{p}=\mathcal{S}(\rho)\left(\widetilde{\sigma}-\hat{\sigma}-\nu \widetilde{\beta}, \frac{1}{3} \overrightarrow{\mathcal{B}}(\rho)(\widetilde{\sigma}-\hat{\sigma}-\nu \widetilde{\beta}),-\gamma \mathcal{K}(\rho) \overrightarrow{\mathcal{N}}(\rho)\right),
\end{array}\right.
$$

we have that $(\widetilde{\sigma}, \widetilde{\beta}, \widetilde{\mathbf{v}}, \widetilde{p}, \rho)$ is a solution of problem (2.8)-(2.18). 
Note that (3.10) is a fully non-linear problem. In the sequel, we shall prove that it is of parabolic type and establish the local well-posedness by applying analytic semigroup theory. Given two Banach spaces $E_{0}$ and $E_{1}$, such that $E_{1}$ is continuously and densely embedded into $E_{0}$, we denote by $\mathcal{H}\left(E_{1}, E_{0}\right)$ the subset of all linear operators $A \in L\left(E_{1}, E_{0}\right)$ such that $-A$ generates a strongly continuous analytic semigroup on $E_{0}$. We denote by $D$ the Fréchet derivative of smooth operators from $\mathcal{O}$ to $B_{q q}^{3-1 / q}\left(\mathbb{S}^{2}\right)$. By a slight modification of the proof of Lemma 2.6 and Corollary 1 in [28], we get the following fundamental result:

Lemma 3.4. (i) $-D \mathcal{Q}(0) \in \mathcal{H}\left(B_{q q}^{4-1 / q}\left(\mathbb{S}^{2}\right), B_{q q}^{3-1 / q}\left(\mathbb{S}^{2}\right)\right)$.

(ii) Let $a_{0}>0$ be sufficiently small. Then for any $\rho \in \mathcal{O}$ there holds

$$
-D \mathcal{Q}(\rho) \in \mathcal{H}\left(B_{q q}^{4-1 / q}\left(\mathbb{S}^{2}\right), B_{q q}^{3-1 / q}\left(\mathbb{S}^{2}\right)\right)
$$

Lemma 3.4 implies that problem (3.10) is of the parabolic type in $\mathcal{O}$ for sufficiently small $a_{0}$, in the sense of Amann [1] and Lunardi 25]. Thus by analytic semigroup theory and applications to parabolic differential problems (cf. Theorem 8.1.1 and Theorem 8.3.4 in [25]), we get the following local well-posedness result:

Theorem 3.5. Let $a_{0}>0$ be sufficiently small. For any given $\rho_{0} \in \mathcal{O}$, there exists $t^{+}:=t^{+}\left(\rho_{0}\right)$ such that problem (3.10) has a unique solution

$$
\rho:=\rho\left(\cdot, \rho_{0}\right) \in C\left(\left[0, t^{+}\right), \mathcal{O}\right) \cap C^{1}\left(\left[0, t^{+}\right), B_{q q}^{3-1 / q}\left(\mathbb{S}^{2}\right)\right) .
$$

Moreover, the mapping $\left(t, \rho_{0}\right) \mapsto \rho\left(t, \rho_{0}\right)$ defines a smooth semiflow on $\mathcal{O}$.

\section{SpeCtrum AnALYSis}

In this section we study the spectrum of $D \mathcal{Q}(0)$. Since $B_{q q}^{4-1 / q}\left(\mathbb{S}^{2}\right)$ is compactly embedded into $B_{q q}^{3-1 / q}\left(\mathbb{S}^{2}\right)$, it follows from Lemma 3.4 that the spectrum of the operator $D \mathcal{Q}(0)$, which we denote by $\sigma(D \mathcal{Q}(0))$, consists of all eigenvalues.

To compute the eigenvalues of $D \mathcal{Q}(0)$, we need to study the linearization of problem (1.1) -(1.11) at the radial stationary solution $\left(\sigma_{s}, \beta_{s}, \mathbf{v}_{s}, p_{s}, 0\right)$. Consider

$$
\begin{cases}\sigma(t, x)=\sigma_{s}(r)+\varepsilon \phi(t, r, \omega), & \beta(t, x)=\beta_{s}(r)+\varepsilon \varphi(t, r, \omega), \\ \mathbf{v}(t, x)=\mathbf{v}_{s}(x)+\varepsilon \vec{v}(t, r, \omega), & p(t, x)=p_{s}(r)+\varepsilon \psi(t, r, \omega), \\ \Omega(t)=\left\{x \in \mathbb{R}^{3}: r<R_{s}+\varepsilon \eta(t, \omega)\right\} & \left(r=|x|, \omega=\frac{x}{|x|} \in \mathbb{S}^{2}\right),\end{cases}
$$

where $\varepsilon$ is a small real parameter and $\phi, \varphi, \vec{v}, \psi$ and $\eta$ are new unknown functions. Let $\mathbf{e}_{r}(x)=x / r$ be the unit outward normal field on $\partial \Omega_{s}$. Denote by $\Delta_{\omega}$ the Laplace-Beltrami operator on the sphere $\mathbb{S}^{2}$. Substituting (4.1) into (1.1)-(1.10) and collecting all $\varepsilon$-order terms in each equation, we get the linearized problem as 
follows (see Section 4 of [18]):

$$
\begin{array}{cl}
\Delta \phi=\lambda_{1} \phi+\varphi & \text { in } \Omega_{s}, \\
\Delta \varphi=\lambda_{2} \varphi & \text { in } \Omega_{s}, \\
\phi=-\sigma_{s}^{\prime}\left(R_{s}\right) \eta \quad \text { on } \partial \Omega_{s}, \\
\varphi=-\beta_{s}^{\prime}\left(R_{s}\right) \eta \quad \text { on } \partial \Omega_{s}, \\
\nabla \cdot \vec{v}=\phi-\nu \varphi \quad \text { in } \Omega_{s}, \\
-\Delta \vec{v}+\nabla \psi-\frac{1}{3} \nabla(\nabla \cdot \vec{v})=0 \quad \text { in } \Omega_{s}, \\
\mathbf{T}(\vec{v}, \psi) \mathbf{e}_{r}=-\frac{2}{R_{s}}(\bar{\sigma}-\hat{\sigma}-\nu \bar{\beta}) \nabla_{\omega} \eta+\frac{\gamma}{R_{s}^{2}}\left(\eta+\frac{1}{2} \Delta_{\omega} \eta\right) \mathbf{e}_{r} \\
+\frac{4}{R_{s}}(\bar{\sigma}-\hat{\sigma}-\nu \bar{\beta}) \eta \mathbf{e}_{r} \quad \text { on } \partial \Omega_{s}, \\
\partial_{t} \eta=\left.\vec{v}\right|_{r=R_{s}} \cdot \mathbf{e}_{r}+(\bar{\sigma}-\hat{\sigma}-\nu \bar{\beta}) \eta \quad \text { on } \partial \Omega_{s}, \\
\int_{\Omega_{s}} \vec{v} \mathrm{~d} x=0, \\
\int_{\Omega_{s}} \vec{v} \times x \mathrm{~d} x=0 .
\end{array}
$$

As in Section 3, we can reduce equations (4.2)-4.11) into a differential equation containing the unknown function $\eta$ only. Indeed, for given $T>0$ and $\eta \in$ $C\left([0, T], B_{q q}^{4-1 / q}\left(\mathbb{S}^{2}\right)\right)$, we first solve the elliptic boundary value problem (4.2)-4.5) to get a unique solution $(\phi(t, \cdot), \varphi(t, \cdot)) \in W^{4, q}\left(\Omega_{s}\right) \times W^{4, q}\left(\Omega_{s}\right)$ as a functional of $\eta$, and next substitute the obtained $\phi$ and $\varphi$ into (4.6). By using Lemma 3.2 we get a unique solution $(\vec{v}(t, \cdot), \psi(t, \cdot)) \in W^{3, q}\left(\Omega_{s}\right)^{3} \times W^{2, q}\left(\Omega_{s}\right)$ of problem (4.6)-(4.8), (4.10) and (4.11), as a functional of $\eta$. Thus by substituting the solution $\vec{v}(t, \cdot)$ into (4.9) and defining a mapping $\widetilde{\mathcal{Q}}: B_{q q}^{4-1 / q}\left(\mathbb{S}^{2}\right) \rightarrow B_{q q}^{3-1 / q}\left(\mathbb{S}^{2}\right)$ by

$$
\widetilde{\mathcal{Q}}(\eta):=\left.\vec{v}\right|_{r=R_{s}} \cdot \mathbf{e}_{r}+(\bar{\sigma}-\hat{\sigma}-\nu \bar{\beta}) \eta,
$$

we reduce equations (4.2)-(4.11) into the following equation:

$$
\partial_{t} \eta=\widetilde{\mathcal{Q}}(\eta) .
$$

It is easy to see that the linearization of the first equation of (3.10) is given by

$$
\partial_{t} \eta=D \mathcal{Q}(0) \eta \text {. }
$$

By Lemma 2.1 and Lemma 3.3, problem (1.1)-(1.11) is equivalent to problem (3.10). Hence the corresponding linearizations of (1.1)-(1.11) and (3.10) at the radial stationary solution should be equivalent. That is,

Lemma 4.1. $D \mathcal{Q}(0)=\widetilde{\mathcal{Q}}$.

In the following, we study the eigenvalues of the operator $\widetilde{\mathcal{Q}}$. Motivated by 18 and $[28$, we shall see that $\widetilde{\mathcal{Q}}$ can be expressed in terms of Fourier expansions of spherical harmonics. For each $l \in \mathbb{N} \cup\{0\}$, let $Y_{l m}(\omega)(m=-l,-l+1, \cdots, l-1, l)$ be a normalized orthogonal basis of the space of all spherical harmonics of degree $l$. Note that $\left\{Y_{l m}(\omega): l=0,1,2, \cdots ; m=-l,-l+1, \cdots, l-1, l\right\}$ is a normalized orthogonal basis of $L^{2}\left(\mathbb{S}^{2}\right)$ and

$$
\Delta_{\omega} Y_{l m}=-l(l+1) Y_{l m} .
$$


Let $\vec{V}_{l m}(\omega), \vec{X}_{l m}(\omega)$ and $\vec{W}_{l m}(\omega)$ be the corresponding vector spherical harmonics to $Y_{l m}(\omega)$, where $l=0,1,2, \cdots$ and $m=-l,-l+1, \cdots, l-1, l$. All these vector spherical harmonics form a normalized orthogonal basis of $\left(L^{2}\left(\mathbb{S}^{2}\right)\right)^{3}$ (see Appendix A of [18] and [23]). Denote

$$
L_{l}:=\frac{\mathrm{d}^{2}}{\mathrm{~d} r^{2}}+\frac{2}{r} \frac{\mathrm{d}}{\mathrm{d} r}-\frac{l^{2}+l}{r^{2}}, \quad l \geq 0, l \in \mathbb{N} .
$$

For simplicity, we set

$$
\eta:=Y_{l m}(\omega) .
$$

Substitute it into the above linearized problem, and we first solve the boundary value problem (4.2)-(4.5) to get the solution

$$
\phi=c_{l}(r) Y_{l m}(\omega), \quad \varphi=d_{l}(r) Y_{l m}(\omega),
$$

where $c_{l}(r)$ and $d_{l}(r)$ satisfy

$$
\begin{cases}L_{l} c_{l}=\lambda_{1} c_{l}+d_{l}, & L_{l} d_{l}=\lambda_{2} d_{l} \quad \text { for } 0<r<R_{s}, \\ c_{l}\left(R_{s}\right)=-\sigma_{s}^{\prime}\left(R_{s}\right), & d_{l}\left(R_{s}\right)=-\beta_{s}^{\prime}\left(R_{s}\right) .\end{cases}
$$

Recall that the well-known modified Bessel function $I_{m}(r)(m \geq 0)$, which is given by (see [26])

$$
I_{m}(r)=\sum_{k=0}^{\infty} \frac{(r / 2)^{m+2 k}}{k ! \Gamma(m+k+1)}
$$

is the solution of the problem

$$
\left\{\begin{array}{l}
I_{m}^{\prime \prime}(r)+\frac{1}{r} I_{m}^{\prime}(r)-\left(1+\frac{m^{2}}{r^{2}}\right) I_{m}(r)=0 \quad \text { for } r>0, \\
I_{m}(r) \text { bounded at } r \sim 0 .
\end{array}\right.
$$

By (4.16) and (4.18) we obtain

$$
\begin{aligned}
c_{l}(r)= & -\left(\sigma_{s}^{\prime}\left(R_{s}\right)-\frac{\beta_{s}^{\prime}\left(R_{s}\right)}{\lambda_{2}-\lambda_{1}}\right) \frac{R_{s}^{\frac{1}{2}} I_{l+\frac{1}{2}}\left(\sqrt{\lambda}_{1} r\right)}{r^{\frac{1}{2}} I_{l+\frac{1}{2}}\left(\sqrt{\lambda_{1}} R_{s}\right)} \\
& -\frac{\beta_{s}^{\prime}\left(R_{s}\right) R_{s}^{\frac{1}{2}} I_{l+\frac{1}{2}}\left(\sqrt{\lambda}_{2} r\right)}{\left(\lambda_{2}-\lambda_{1}\right) r^{\frac{1}{2}} I_{l+\frac{1}{2}}\left(\sqrt{\lambda}_{2} R_{s}\right)}, \\
d_{l}(r)= & -\frac{\beta_{s}^{\prime}\left(R_{s}\right) R_{s}^{\frac{1}{2}} I_{l+\frac{1}{2}}\left(\sqrt{\lambda}_{2} r\right)}{r^{\frac{1}{2}} I_{l+\frac{1}{2}}\left(\sqrt{\lambda}_{2} R_{s}\right)} .
\end{aligned}
$$

A simple computation shows that

$$
\begin{aligned}
& \sigma_{s}(r)=A_{1} \frac{R_{s}^{\frac{1}{2}} I_{\frac{1}{2}}\left(\sqrt{\lambda}_{1} r\right)}{r^{\frac{1}{2}} I_{\frac{1}{2}}\left(\sqrt{\lambda_{1}} R_{s}\right)}+\left(A_{2}+\nu \bar{\beta}\right) \frac{R_{s}^{\frac{1}{2}} I_{\frac{1}{2}}\left(\sqrt{\lambda}_{2} r\right)}{r^{\frac{1}{2}} I_{\frac{1}{2}}\left(\sqrt{\lambda_{2}} R_{s}\right)}, \\
& \beta_{s}(r)=\frac{\bar{\beta} R_{s}^{\frac{1}{2}} I_{\frac{1}{2}}\left(\sqrt{\lambda}_{2} r\right)}{r^{\frac{1}{2}} I_{\frac{1}{2}}\left(\sqrt{\lambda}_{2} R_{s}\right)},
\end{aligned}
$$


and

$$
\begin{aligned}
& \sigma_{s}^{\prime}\left(R_{s}\right)=A_{1}{\sqrt{\lambda_{1}}}_{1} \frac{I_{\frac{3}{2}}\left(\sqrt{\lambda}_{1} R_{s}\right)}{I_{\frac{1}{2}}\left(\sqrt{\lambda}_{1} R_{s}\right)}+\left(A_{2}+\nu \bar{\beta}\right) \sqrt{\lambda_{2}} \frac{I_{\frac{3}{2}}\left(\sqrt{\lambda_{2}} R_{s}\right)}{I_{\frac{1}{2}}\left(\sqrt{\left.\lambda_{2} R_{s}\right)}\right.}, \\
& \beta_{s}^{\prime}\left(R_{s}\right)=\bar{\beta}{\sqrt{\lambda_{2}}}_{\frac{I_{\frac{3}{2}}}{}\left(\sqrt{\lambda}_{2} R_{s}\right)}^{I_{\frac{1}{2}}\left(\sqrt{\lambda}_{2} R_{s}\right)} .
\end{aligned}
$$

Note that $A_{1}=\bar{\sigma}-\left[\bar{\beta} /\left(\lambda_{2}-\lambda_{1}\right)\right], A_{2}=\left[\bar{\beta} /\left(\lambda_{2}-\lambda_{1}\right)\right]-\nu \bar{\beta}$. From (4.19) and (4.21) we have

$$
\begin{aligned}
& z_{l}(r):=c_{l}(r)-\nu d_{l}(r) \\
& =-\left[A_{1} \frac{I_{\frac{3}{2}}\left(\sqrt{\lambda}_{1} R_{s}\right)}{I_{\frac{1}{2}}\left(\sqrt{\lambda}_{1} R_{s}\right)} \frac{\lambda_{1}^{\frac{1}{2}} R_{s}^{\frac{1}{2}} I_{l+\frac{1}{2}}\left(\sqrt{\lambda}_{1} r\right)}{r^{\frac{1}{2}} I_{l+\frac{1}{2}}\left(\sqrt{\lambda}_{1} R_{s}\right)}+A_{2} \frac{I_{\frac{3}{2}}\left(\sqrt{\lambda_{2}} R_{s}\right)}{I_{\frac{1}{2}}\left(\sqrt{\lambda_{2}} R_{s}\right)} \frac{\lambda_{2}^{\frac{1}{2}} R_{s}^{\frac{1}{2}} I_{l+\frac{1}{2}}\left(\sqrt{\lambda}_{2} r\right)}{r^{\frac{1}{2}} I_{l+\frac{1}{2}}\left(\sqrt{\lambda}_{2} R_{s}\right)}\right] .
\end{aligned}
$$

Substituting (4.15) into (4.6) we see that

$$
\phi-\nu \varphi=z_{l}(r) Y_{l m}(\omega)
$$

Then problem (4.6)-(4.8), 4.10) and (4.11) reads as

$$
\left\{\begin{array}{l}
\nabla \cdot \vec{v}=z_{l}(r) Y_{l m}(\omega) \\
-\Delta \vec{v}+\nabla \psi-\frac{1}{3} \nabla(\nabla \cdot \vec{v})=0 \\
\left.\mathbf{T}(\vec{v}, \psi)\right|_{r=R_{s}} \mathbf{e}_{r}=-\frac{2}{R_{s}}((\bar{\sigma}-\hat{\sigma})-\nu \bar{\beta}) \nabla_{\omega} Y_{l m}(\omega) \\
\quad+\left[\frac{\gamma}{R_{s}^{2}}\left(1-\frac{l^{2}+l}{2}\right)+\frac{4}{R_{s}}((\bar{\sigma}-\hat{\sigma})-\nu \bar{\beta})\right] Y_{l m}(\omega) \mathbf{e}_{r} \\
\int_{\Omega_{s}} \vec{v} \mathrm{~d} x=0, \quad \int_{\Omega_{s}} \vec{v} \times x \mathrm{~d} x=0 .
\end{array}\right.
$$

The first two equations, (4.23) $)_{1}$ and (4.23) 2 , imply that

$$
\begin{aligned}
& \Delta\left(\psi-\frac{4}{3} z_{l}(r) Y_{l m}(\omega)\right)=0, \\
& \Delta \vec{v}-\nabla\left(z_{l}(r) Y_{l m}(\omega)\right)=\nabla\left(\psi-\frac{4}{3} z_{l}(r) Y_{l m}(\omega)\right) .
\end{aligned}
$$

Thus the solution of problem (4.23) can be expressed by

$$
\begin{aligned}
& \psi(r, \omega)=\frac{4}{3} z_{l}(r) Y_{l m}(\omega)+P_{l m}(r) Y_{l m}(\omega), \\
& \vec{v}(r, \omega)=\mathbf{a}+\mathbf{b} \times x+v_{l m}(r) \vec{V}_{l m}(\omega)+x_{l m}(r) \vec{X}_{l m}(\omega)+w_{l m}(r) \vec{W}_{l m}(\omega),
\end{aligned}
$$

where $\mathbf{a}, \mathbf{b}$ are unknown constant vectors, and $P_{l m}(r), v_{l m}(r), x_{l m}(r)$ and $w_{l m}(r)$ are unknown functions. By the well-known properties of vector spherical harmonics 
(see Appendix A of [18] and 23]), we have

$$
\begin{aligned}
& \nabla\left(z_{l} Y_{l m}\right)=\left[-z_{l}^{\prime}+\frac{l}{r} z_{l}\right] \sqrt{\frac{l+1}{2 l+1}} \vec{V}_{l m}+\left[z_{l}^{\prime}+\frac{l+1}{r} z_{l}\right] \sqrt{\frac{l}{2 l+1}} \vec{W}_{l m}, \\
& \nabla\left(\psi-\frac{4}{3} z_{l} Y_{l m}\right)=\left[-P_{l m}^{\prime}+\frac{l}{r} P_{l m}\right] \sqrt{\frac{l+1}{2 l+1}} \vec{V}_{l m}+\left[P_{l m}^{\prime}+\frac{l+1}{r} P_{l m}\right] \sqrt{\frac{l}{2 l+1}} \vec{W}_{l m}, \\
& \nabla \cdot \vec{v}=-\left[v_{l m}^{\prime}+\frac{l+2}{r} v_{l m}\right] \sqrt{\frac{l+1}{2 l+1}} Y_{l m}+\left[w_{l m}^{\prime}-\frac{l-1}{r} w_{l m}\right] \sqrt{\frac{l}{2 l+1}} Y_{l m}, \\
& \Delta \vec{v}=L_{l+1}\left(v_{l m}\right) \vec{V}_{l m}+L_{l}\left(x_{l m}\right) \vec{X}_{l m}+L_{l-1}\left(w_{l m}\right) \vec{W}_{l m} .
\end{aligned}
$$

Substituting (4.25) and (4.26) into (4.23) 1 and (4.24), we obtain the following ODE system:

$$
\left\{\begin{array}{l}
L_{l}\left(P_{l m}(r)\right)=0, \quad L_{l}\left(x_{l m}(r)\right)=0, \\
-\left[v_{l m}^{\prime}(r)+\frac{l+2}{r} v_{l m}(r)\right] \sqrt{\frac{l+1}{2 l+1}}+\left[w_{l m}^{\prime}(r)-\frac{l-1}{r} w_{l m}(r)\right] \sqrt{\frac{l}{2 l+1}}=z_{l}(r), \\
{\left[-P_{l m}^{\prime}(r)+\frac{l}{r} P_{l m}(r)\right] \sqrt{\frac{l+1}{2 l+1}}=L_{l+1}\left(v_{l m}(r)\right)-\left[-z_{l}^{\prime}(r)+\frac{l}{r} z_{l}(r)\right] \sqrt{\frac{l+1}{2 l+1}},} \\
{\left[P_{l m}^{\prime}(r)+\frac{l+1}{r} P_{l m}(r)\right] \sqrt{\frac{l}{2 l+1}}=L_{l-1}\left(w_{l m}(r)\right)-\left[z_{l}^{\prime}(r)+\frac{l+1}{r} z_{l}(r)\right] \sqrt{\frac{l}{2 l+1}} .}
\end{array}\right.
$$

We define

$$
\begin{aligned}
& y_{l}(r):=A_{1} \frac{I_{\frac{3}{2}}\left(\sqrt{\lambda_{1}} R_{s}\right)}{I_{\frac{1}{2}}\left(\sqrt{\lambda_{1}} R_{s}\right)} \frac{R_{s}^{\frac{1}{2}} I_{l+\frac{3}{2}}\left(\sqrt{\lambda_{1}} r\right)}{r^{\frac{1}{2}} I_{l+\frac{1}{2}}\left(\sqrt{\lambda_{1}} R_{s}\right)}+A_{2} \frac{I_{\frac{3}{2}}\left(\sqrt{\lambda_{2}} R_{s}\right)}{I_{\frac{1}{2}}\left(\sqrt{\lambda_{2}} R_{s}\right)} \frac{R_{s}^{\frac{1}{2}} I_{l+\frac{3}{2}}\left(\sqrt{\lambda}_{2} r\right)}{r^{\frac{1}{2}} I_{l+\frac{1}{2}}\left(\sqrt{\lambda_{2}} R_{s}\right)}, \\
& \tilde{y}_{l}(r):=A_{1} \frac{I_{\frac{3}{2}}\left(\sqrt{\lambda_{1}} R_{s}\right)}{I_{\frac{1}{2}}\left(\sqrt{\lambda_{1}} R_{s}\right)} \frac{R_{s}^{\frac{1}{2}} I_{l-\frac{1}{2}}\left(\sqrt{\lambda_{1}} r\right)}{r^{\frac{1}{2}} I_{l+\frac{1}{2}}\left(\sqrt{\lambda_{1}} R_{s}\right)}+A_{2} \frac{I_{\frac{3}{2}}\left(\sqrt{\lambda_{2}} R_{s}\right)}{I_{\frac{1}{2}}\left(\sqrt{\lambda_{2}} R_{s}\right)} \frac{R_{s}^{\frac{1}{2}} I_{l-\frac{1}{2}}\left(\sqrt{\lambda}_{2} r\right)}{r^{\frac{1}{2}} I_{l+\frac{1}{2}}\left(\sqrt{\lambda}_{2} R_{s}\right)} .
\end{aligned}
$$

Lemma 4.2. The general solutions of the ODE system (4.27) have the expressions as

$$
\left\{\begin{array}{l}
P_{l m}(r)=2(2 l+3) B_{1} r^{l}, \quad x_{l m}(r)=C_{1} r^{l}, \\
v_{l m}(r)=\sqrt{\frac{l+1}{2 l+1}} \frac{2 l}{l+1} B_{1} r^{l+1}+\sqrt{\frac{l+1}{2 l+1}} y_{l}(r), \\
w_{l m}(r)=D_{1} r^{l-1}+\sqrt{\frac{l}{2 l+1}}(2 l+3) B_{1} r^{l+1}-\sqrt{\frac{l}{2 l+1}}\left[\tilde{y}_{l}(r)-\tilde{y}_{l}\left(R_{s}\right) \frac{r^{l-1}}{R_{s}^{l-1}}\right],
\end{array}\right.
$$

where $B_{1}, C_{1}$ and $D_{1}$ are constants, and $y_{l}(r), \tilde{y}_{l}(r)$ are given by (4.28).

Proof. It is not difficult to verify that the functions

$$
\begin{aligned}
& P_{l m}(r)=2(2 l+3) B_{1} r^{l}, \quad x_{l m}(r)=C_{1} r^{l}, \\
& v_{l m}(r)=\sqrt{\frac{l+1}{2 l+1}} \frac{2 l}{l+1} B_{1} r^{l+1}-\sqrt{\frac{l+1}{2 l+1}} r^{-l-2} \int_{0}^{r} s^{l+2} z_{l}(s) \mathrm{d} s, \\
& w_{l m}(r)=D_{1} r^{l-1}+\sqrt{\frac{l}{2 l+1}}(2 l+3) B_{1} r^{l+1}-\sqrt{\frac{l}{2 l+1}} r^{l-1} \int_{r}^{R_{s}} s^{-l+1} z_{l}(s) \mathrm{d} s
\end{aligned}
$$


satisfy the ODE system (4.27), where $B_{1}, C_{1}$ and $D_{1}$ are constants. Recall the well-known formulas for modified Bessel functions (see [26]):

$$
\frac{\mathrm{d}}{\mathrm{d} r}\left(r^{m} I_{m}(r)\right)=r^{m} I_{m-1}(r) \quad \text { and } \quad \frac{\mathrm{d}}{\mathrm{d} r}\left(r^{-m} I_{m}(r)\right)=r^{-m} I_{m+1}(r) .
$$

By the above formulas we easily have

$$
r^{-l-2} \int_{0}^{r} \sqrt{\lambda}_{1} s^{l+\frac{3}{2}} I_{l+\frac{1}{2}}\left(\sqrt{\lambda}_{1} s\right) \mathrm{d} s=r^{-\frac{1}{2}} I_{l+\frac{3}{2}}\left(\sqrt{\lambda}_{1} r\right) .
$$

Then by (4.22) and (4.28), we immediately get

$$
r^{-l-2} \int_{0}^{r} s^{l+2} z_{l}(s) \mathrm{d} s=-y_{l}(r) .
$$

Hence,

$$
v_{l m}(r)=\sqrt{\frac{l+1}{2 l+1}} \frac{2 l}{l+1} B_{1} r^{l+1}+\sqrt{\frac{l+1}{2 l+1}} y_{l}(r) .
$$

Similarly, we get

$$
w_{l m}(r)=D_{1} r^{l-1}+\sqrt{\frac{l}{2 l+1}}(2 l+3) B_{1} r^{l+1}-\sqrt{\frac{l}{2 l+1}}\left[\tilde{y}_{l}(r)-\tilde{y}_{l}\left(R_{s}\right) \frac{r^{l-1}}{R_{s}^{l-1}}\right] .
$$

The proof is completed.

Next we consider (4.23) 3 , i.e., the third equation of (4.23). Recall the following two well-known formulas (see Appendix A of [18] or [23]):

$$
\begin{array}{r}
\nabla_{\omega} Y_{l m}(\omega)=l \sqrt{\frac{l+1}{2 l+1}} \vec{V}_{l m}(\omega)+(l+1) \sqrt{\frac{l}{2 l+1}} \vec{W}_{l m}(\omega), \\
Y_{l m}(\omega) \mathbf{e}_{r}=-\sqrt{\frac{l+1}{2 l+1}} \vec{V}_{l m}(\omega)+\sqrt{\frac{l}{2 l+1}} \vec{W}_{l m}(\omega) .
\end{array}
$$

We rewrite $\vec{v}$ in (4.25) as

$$
\vec{v}(r, \omega)=\mathbf{a}+\mathbf{b} \times x+x_{l m}(r) \vec{X}_{l m}(\omega)+H_{1}(r) Y_{l m}(\omega) \mathbf{e}_{r}+H_{2}(r) \nabla_{\omega} Y_{l m}(\omega),
$$

where

$$
\begin{aligned}
& H_{1}(r)=-\sqrt{\frac{l+1}{2 l+1}} v_{l m}(r)+\sqrt{\frac{l}{2 l+1}} w_{l m}(r), \\
& H_{2}(r)=\frac{v_{l m}(r)}{\sqrt{(l+1)(2 l+1)}}+\frac{w_{l m}(r)}{\sqrt{l(2 l+1)}} .
\end{aligned}
$$

By Lemma 4.4 of [18] and [23], a direct computation shows that

$\left[\nabla \otimes\left(H_{1}(r) Y_{l m} \mathbf{e}_{r}\right)+\left(\nabla \otimes\left(H_{1}(r) Y_{l m} \mathbf{e}_{r}\right)\right)^{T}\right] \cdot \mathbf{e}_{r}=2 H_{1}^{\prime}(r) Y_{l m} \mathbf{e}_{r}+r^{-1} H_{1}(r) \nabla_{\omega} Y_{l m}$,

$\left[\nabla \otimes\left(H_{2}(r) \nabla_{\omega} Y_{l m}\right)+\left(\nabla \otimes\left(H_{2}(r) \nabla_{\omega} Y_{l m}\right)\right)^{T}\right] \cdot \mathbf{e}_{r}=\left(H_{2}^{\prime}(r)-r^{-1} H_{2}(r)\right) \nabla_{\omega} Y_{l m}$,

$\left[\nabla \otimes\left(x_{l m}(r) \vec{X}_{l m}\right)+\left(\nabla \otimes\left(x_{l m}(r) \vec{X}_{l m}\right)\right)^{T}\right] \cdot \mathbf{e}_{r}=\left(x_{l m}^{\prime}(r)-r^{-1} x_{l m}\right) \vec{X}_{l m}$. 
Thus by (4.25), (4.29) (4.31) and Lemma 4.2, we have

$$
\begin{aligned}
\left.\mathbf{T}(\vec{v}, \psi)\right|_{r=R_{s}} \mathbf{e}_{r}= & {\left[\nabla \otimes \vec{v}+(\nabla \otimes \vec{v})^{T}-\left(\psi+\frac{2}{3} \nabla \cdot \vec{v}\right) \mathbf{I}\right]_{r=R_{s}} \mathbf{e}_{r} } \\
= & C_{1}(l-1) R_{s}^{l-1} \vec{X}_{l m}+2\left[H_{1}^{\prime}\left(R_{s}\right)-z_{l}\left(R_{s}\right)-(2 l+3) B_{1} R_{s}^{l}\right] Y_{l m} \mathbf{e}_{r} \\
& +R_{s}^{-1}\left[H_{1}\left(R_{s}\right)-H_{2}\left(R_{s}\right)+R_{s} H_{2}^{\prime}\left(R_{s}\right)\right] \nabla_{\omega} Y_{l m} .
\end{aligned}
$$

Since $\vec{X}_{l m}, Y_{l m} \mathbf{e}_{r}$ and $\nabla_{\omega} Y_{l m}$ are mutually orthogonal, by combining (4.23) 3 and (4.32), we immediately get

$$
C_{1}(l-1) R_{s}^{l-1}=0
$$

$$
\begin{gathered}
2\left[H_{1}^{\prime}\left(R_{s}\right)-z_{l}\left(R_{s}\right)-(2 l+3) B_{1} R_{s}^{l}\right]=\frac{\gamma}{R_{s}^{2}}\left(1-\frac{l^{2}+l}{2}\right)+\frac{4}{R_{s}}((\bar{\sigma}-\hat{\sigma})-\nu \bar{\beta}), \\
H_{1}\left(R_{s}\right)-H_{2}\left(R_{s}\right)+R_{s} H_{2}^{\prime}\left(R_{s}\right)=-2((\bar{\sigma}-\hat{\sigma})-\nu \bar{\beta}) .
\end{gathered}
$$

It follows from (4.33) that $C_{1}=0$. By (4.28), (4.30) and Lemma 4.2, we have

$$
\begin{aligned}
& H_{1}\left(R_{s}\right)=l B_{1} R_{s}^{l+1}+l \tilde{D}_{1} R_{s}^{l+1}-\frac{l+1}{2 l+1} y_{l}\left(R_{s}\right) \\
& H_{2}\left(R_{s}\right)=\frac{l+3}{l+1} B_{1} R_{s}^{l+1}+\tilde{D}_{1} R_{s}^{l+1}+\frac{1}{(2 l+1) R_{s}^{l+2}} y_{l}\left(R_{s}\right), \\
& H_{1}^{\prime}\left(R_{s}\right)=l(l+1) B_{1} R_{s}^{l}+l(l-1) \tilde{D}_{1} R_{s}^{l}+z_{l}\left(R_{s}\right)+\frac{(l+2)(l+1)}{(2 l+1) R_{s}} y_{l}\left(R_{s}\right), \\
& H_{2}^{\prime}\left(R_{s}\right)=(l+3) B_{1} R_{s}^{l}+(l-1) \tilde{D}_{1} R_{s}^{l}-\frac{l+2}{(2 l+1) R_{s}} y_{l}\left(R_{s}\right),
\end{aligned}
$$

where $\tilde{D}_{1}=D_{1} /\left(\sqrt{l(2 l+1)} R_{s}^{2}\right)$ and

$$
y_{l}\left(R_{s}\right)=A_{1} \frac{I_{\frac{3}{2}}\left(\sqrt{\lambda_{1}} R_{s}\right)}{I_{\frac{1}{2}}\left(\sqrt{\lambda_{1}} R_{s}\right)} \frac{I_{l+\frac{3}{2}}\left(\sqrt{\lambda}_{1} R_{s}\right)}{I_{l+\frac{1}{2}}\left(\sqrt{\lambda}_{1} R_{s}\right)}+A_{2} \frac{I_{\frac{3}{2}}\left(\sqrt{\lambda_{2}} R_{s}\right)}{I_{\frac{1}{2}}\left(\sqrt{\lambda_{2}} R_{s}\right)} \frac{I_{l+\frac{3}{2}}\left(\sqrt{\lambda}_{2} R_{s}\right)}{I_{l+\frac{1}{2}}\left(\sqrt{\lambda_{2}} R_{s}\right)} .
$$

Substituting (4.36) into (4.34) and (4.35), we get

$$
\begin{gathered}
\left(l^{2}-l-3\right) B_{1} R_{s}^{l}+l(l-1) \tilde{D}_{1} R_{s}^{l}+\frac{(l+2)(l+1)}{(2 l+1) R_{s}} y_{l}\left(R_{s}\right) \\
=\frac{\gamma}{4 R_{s}^{2}}\left(2-l^{2}-l\right)+\frac{2}{R_{s}}((\bar{\sigma}-\hat{\sigma})-\nu \bar{\beta}), \\
\frac{l(l+2)}{l+1} B_{1} R_{s}^{l+1}+(l-1) \tilde{D}_{1} R_{s}^{l+1}-\frac{l+2}{(2 l+1)} y_{l}\left(R_{s}\right)=-(\bar{\sigma}-\hat{\sigma})+\nu \bar{\beta} .
\end{gathered}
$$

Due to the condition (4.10) we have (cf. (5.8) in [18])

(4.40) $\mathbf{a}=-\frac{3}{4 \pi} \int_{\Omega_{s}}\left\{H_{1}(r) Y_{l m}(\omega) \omega+H_{2}(r) \nabla_{\omega} Y_{l m}(\omega)\right\} \mathrm{d} x=0 \quad$ for $l \in \mathbb{N}, l \neq 1$. 
Note that $(\mathbf{b} \times x) \cdot \mathbf{e}_{r}=0$ and $\nabla_{\omega} Y_{l m}(\omega) \cdot \mathbf{e}_{r}=0$. Thus by Lemma 4.1, (4.29) and (4.36) we have

$$
\begin{aligned}
& D \mathcal{Q}(0) Y_{l m}(\omega) \\
= & \left.\vec{v}\right|_{r=R_{s}} \cdot \mathbf{e}_{r}+(\bar{\sigma}-\hat{\sigma}-\nu \bar{\beta}) Y_{l m}(\omega) \\
= & \left(\mathbf{a}+\mathbf{b} \times x+H_{2}\left(R_{s}\right) \nabla_{\omega} Y_{l m}(\omega)\right) \cdot \mathbf{e}_{r}+\left(H_{1}\left(R_{s}\right)+\bar{\sigma}-\hat{\sigma}-\nu \bar{\beta}\right) Y_{l m}(\omega) \\
= & \left\{l\left(B_{1}+\tilde{D}_{1}\right) R_{s}^{l+1}-\frac{l+1}{(2 l+1)} y_{l}\left(R_{s}\right)+\bar{\sigma}-\hat{\sigma}-\nu \bar{\beta}\right\} Y_{l m}(\omega) .
\end{aligned}
$$

By computing (4.38) $\left.\times(2 l+1) R_{s}+4.39\right) \times 3(l+1)$, for $l \neq 1$, we have

$$
\begin{aligned}
\left(B_{1}+\tilde{D}_{1}\right) R_{s}^{l+1}= & \frac{1}{2 l^{2}+4 l+3}\left\{-\frac{\gamma}{4 R_{s}}(2 l+1)(l+2)+\bar{\sigma}-\hat{\sigma}-\nu \bar{\beta}\right. \\
& \left.-\frac{2(l+1)(l+2)}{(2 l+1)} y_{l}\left(R_{s}\right)\right\} .
\end{aligned}
$$

Hence, for $l \neq 1$,

$$
\begin{aligned}
D \mathcal{Q}(0) Y_{l m}(\omega)= & \frac{Y_{l m}(\omega)}{2 l^{2}+4 l+3}\{(l+1)(2 l+3)(\bar{\sigma}-\hat{\sigma}-\nu \bar{\beta}) \\
& \left.-\frac{\gamma}{4 R_{s}} l(l+2)(2 l+1)-(l+1)(2 l+3) y_{l}\left(R_{s}\right)\right\} .
\end{aligned}
$$

We define

$$
\gamma_{l}:=\frac{4(l+1)(2 l+3)}{l(l+2)(2 l+1)} R_{s}\left(\bar{\sigma}-\hat{\sigma}-\nu \bar{\beta}-y_{l}\left(R_{s}\right)\right) \quad \text { for } \quad l \in \mathbb{N}, l \geq 2,
$$

where $y_{l}\left(R_{s}\right)$ is given by (4.37). Denote

$$
\begin{aligned}
& \alpha_{0}(\gamma):=\bar{\sigma}-\hat{\sigma}-\nu \bar{\beta}-y_{0}\left(R_{s}\right), \\
& \alpha_{l}(\gamma):=-\frac{l(l+2)(2 l+1)}{4\left(2 l^{2}+4 l+3\right) R_{s}}\left(\gamma-\gamma_{l}\right), \quad l \geq 2 .
\end{aligned}
$$

Then (4.42) can be rewritten as follows:

$$
D \mathcal{Q}(0) Y_{l m}(\omega)=\alpha_{l}(\gamma) Y_{l m}(\omega) \quad \text { for } \quad l \neq 1,-l \leq m \leq l .
$$

Finally, we consider the case $l=1$. Since problem (1.1)-(1.11) is translation invariant, by similar arguments as those in [9] and [18, we have

$$
D \mathcal{Q}(0) Y_{1 m}(\omega)=0, \quad m=-1,0,1 .
$$

In summary, we have proved that

Lemma 4.3. $D \mathcal{Q}(0)$ is a Fourier multiplication operator having the following expression: For any $\eta \in C^{\infty}\left(\mathbb{S}^{2}\right)$ with Fourier expansion $\eta(\omega)=\sum_{l=0}^{\infty} \sum_{m=-l}^{l} c_{l m} Y_{l m}(\omega)$,

$$
D \mathcal{Q}(0) \eta(\omega)=\sum_{l \neq 1} \sum_{m=-l}^{l} \alpha_{l}(\gamma) c_{l m} Y_{l m}(\omega)
$$

where $\alpha_{l}(\gamma)$ is given by (4.44). 
As mentioned at the beginning of this section, the spectrum $\sigma(D \mathcal{Q}(0))$ of $D \mathcal{Q}(0)$ consists only of eigenvalues. Thus by Lemma 4.3 we have the following result:

Lemma 4.4. (i) $\sigma(D \mathcal{Q}(0))=\{0\} \cup\left\{\alpha_{l}(\gamma)\right\}_{l \neq 1}$.

(ii) If $\gamma>0$ and $\gamma \notin\left\{\gamma_{l}\right\}_{l \geq 2}$, then the multiplicity of the eigenvalue 0 is 3 .

Next we study the sign of the eigenvalue $\alpha_{l}(r)$. We need the following properties of $\left\{\gamma_{l}\right\}_{l \geq 2}$.

Lemma 4.5. (i) $\lim _{l \rightarrow \infty} \gamma_{l}=0$.

(ii) If $\bar{\sigma}-\hat{\sigma}-\nu \bar{\beta}>0$, then there exists a positive integer $l^{*}$ such that $\gamma_{l}$ is positive and monotone decreasing for $l \geq l^{*}$.

(iii) If $\bar{\sigma}-\hat{\sigma}-\nu \bar{\beta} \leq 0$, then there are at most finite many positive $\gamma_{l}$. In particular, if $\lambda_{2} \geq \lambda_{1}+(1 / \nu)$ and $\bar{\beta} \geq\left(\lambda_{2}-\lambda_{1}\right) \bar{\sigma}$, then for every $l \geq 2$, we have $\gamma_{l} \leq 0$

(iv) For every $l \geq 2, \gamma_{l}$ is monotone decreasing for $\bar{\beta}>0$.

Proof. (i) By the well-known formula (see [26])

$$
I_{l}(r)=\sqrt{\frac{1}{2 \pi l}}\left(\frac{\mathrm{e} r}{2 l}\right)^{l}\left(1+O\left(\frac{1}{l}\right)\right) \text { as } l \rightarrow \infty,
$$

we have

$$
\frac{I_{l+\frac{3}{2}}(r)}{I_{l+\frac{1}{2}}(r)}=\frac{(2 l+1)^{l+1}}{(2 l+3)^{l+2}}\left(1+O\left(\frac{1}{l}\right)\right) \mathrm{e} r=\frac{r}{2 l}\left(1+O\left(\frac{1}{l}\right)\right), \quad \text { as } l \rightarrow \infty .
$$

Combining (4.37), (4.43) and (4.47), we get

$$
\gamma_{l}=\frac{4(l+1)(2 l+3)}{l(l+2)(2 l+1)} R_{s}\left(\bar{\sigma}-\hat{\sigma}-\nu \bar{\beta}-\frac{C}{2 l}+O\left(\frac{1}{l^{2}}\right)\right), \quad \text { as } l \rightarrow \infty,
$$

where $C=\sqrt{\lambda_{1}} A_{1} R_{s} \frac{I_{\frac{3}{2}}\left(\sqrt{\lambda_{1}} R_{s}\right)}{I_{\frac{1}{2}}\left(\sqrt{\lambda_{1}} R_{s}\right)}+\sqrt{\lambda_{2}} A_{2} R_{s} \frac{I_{\frac{3}{2}}\left(\sqrt{\lambda_{2}} R_{s}\right)}{I_{\frac{1}{2}}\left(\sqrt{\lambda_{2}} R_{s}\right)}$. Hence, we have $\lim _{l \rightarrow \infty} \gamma_{l}=$ 0 .

(ii) By (4.48), we see that if $\bar{\sigma}-\hat{\sigma}-\nu \bar{\beta}>0$, then $\gamma_{l}>0$ for $l$ large enough, and if $\bar{\sigma}-\hat{\sigma}-\nu \bar{\beta} \leq 0$, then $\gamma_{l} \leq 0$ for $l$ large enough. A direct computation shows that

$\gamma_{l+1}-\gamma_{l}=-(\bar{\sigma}-\hat{\sigma}-\nu \bar{\beta}) \cdot \frac{4 R_{s}\left(4 l^{4}+28 l^{3}+73 l^{2}+79 l+27\right)}{l(l+1)(l+2)(l+3)(2 l+1)(2 l+3)}+O\left(\frac{1}{l^{3}}\right), \quad$ as $\quad l \rightarrow \infty$.

Thus assertion (ii) follows readily from (4.48) and (4.49).

(iii) Note that

$$
g(r)=\frac{(r \operatorname{coth} r-1)}{r^{2}}=\frac{I_{\frac{3}{2}}(r)}{r I_{\frac{1}{2}}(r)} .
$$

Using this formula and (1.18), we have

$$
f\left(R_{s}\right)=A_{1} \frac{I_{\frac{3}{2}}\left(\sqrt{\lambda_{1}} R_{s}\right)}{\sqrt{\lambda_{1}} R_{s} I_{\frac{1}{2}}\left(\sqrt{\lambda_{1}} R_{s}\right)}+A_{2} \frac{I_{\frac{3}{2}}\left(\sqrt{\lambda_{2}} R_{s}\right)}{\sqrt{\lambda_{2}} R_{s} I_{\frac{1}{2}}\left(\sqrt{\lambda_{2}} R_{s}\right)}=\frac{1}{3} \hat{\sigma} .
$$

Since $A_{1}=\bar{\sigma}-\left[\bar{\beta} /\left(\lambda_{2}-\lambda_{1}\right)\right]$ and $A_{2}=\left[\bar{\beta} /\left(\lambda_{2}-\lambda_{1}\right)\right]-\nu \bar{\beta}$, we have $A_{1}+A_{2}=\bar{\sigma}-\nu \bar{\beta}$. Then we can rewrite (4.43) as

$$
\gamma_{l}=\frac{4(l+1)(2 l+3)}{l(l+2)(2 l+1)} R_{s}\left(A_{1} G_{l}\left(\sqrt{\lambda}_{1} R_{s}\right)+A_{2} G_{l}\left(\sqrt{\lambda}_{2} R_{s}\right)\right)
$$


where

$$
G_{l}(r):=1-\frac{3 I_{\frac{3}{2}}(r)}{r I_{\frac{1}{2}}(r)}-\frac{I_{\frac{3}{2}}(r) I_{l+\frac{3}{2}}(r)}{I_{\frac{1}{2}}(r) I_{l+\frac{1}{2}}(r)}, \quad r>0 .
$$

By (4.46) of [27], we have that $G_{l}(r)>0$ for all $l \geq 2$. If $\lambda_{2} \geq \lambda_{1}+(1 / \nu)$ and $\bar{\beta} \geq\left(\lambda_{2}-\lambda_{1}\right) \bar{\sigma}$, then $A_{1} \leq 0$ and $A_{2} \leq 0$. Thus $\gamma_{l} \leq 0$ for all $l \geq 2$, and the proof of (iii) is completed.

(iv) Assertion (iv) follows similarly from the proof of Lemma 6.1 of [27, with slight modifications.

Define

$$
\gamma_{*}:=\sup \left\{0, \gamma_{2}, \gamma_{3}, \cdots\right\} .
$$

From Lemma 4.5 we know that (4.54) is well-defined and there holds

Lemma 4.6. (i) If $\bar{\sigma}-\hat{\sigma}-\nu \bar{\beta}>0$, then $0<\gamma_{*}<\infty$.

(ii) If $\lambda_{2} \geq \lambda_{1}+(1 / \nu)$ and $\bar{\beta} \geq\left(\lambda_{2}-\lambda_{1}\right) \bar{\sigma}$, then $\gamma_{*}=0$.

(iii) $\gamma_{*}$ is monotone decreasing for $\bar{\beta}>0$.

Denote

$$
\begin{aligned}
\sigma_{+}(D \mathcal{Q}(0)) & :=\{\lambda \in \sigma(D \mathcal{Q}(0)): \operatorname{Re} \lambda>0\}, \\
\sigma_{-}(D \mathcal{Q}(0)) & :=\{\lambda \in \sigma(D \mathcal{Q}(0)): \operatorname{Re} \lambda<0\} .
\end{aligned}
$$

With the above preparations, we have the following assertions:

Lemma 4.7. (i) If $f^{\prime}\left(R_{s}\right)<0$, then $\alpha_{0}(\gamma)<0$. If $f^{\prime}\left(R_{s}\right)>0$, then $\alpha_{0}(\gamma)>0$.

(ii) If $f^{\prime}\left(R_{s}\right)<0$ and $\gamma>\gamma_{*}$, then $\sigma_{+}(D \mathcal{Q}(0))=\emptyset$.

(iii) If $f^{\prime}\left(R_{s}\right)>0$ or $0<\gamma<\gamma_{*}$, then $\sigma_{+}(D \mathcal{Q}(0)) \neq \emptyset$.

Proof. (i) Recall the following well-known formulas (see [26]):

$$
\begin{aligned}
& I_{m}^{\prime}(r)+\frac{m}{r} I_{m}(r)=I_{m-1}(r) \quad \text { for } m \geq 1, \\
& I_{m}^{\prime}(r)-\frac{m}{r} I_{m}(r)=I_{m+1}(r) \quad \text { for } m \geq 0 .
\end{aligned}
$$

From (4.50), (4.53) and (4.55) we get

$$
G_{0}(r)=r g^{\prime}(r), \quad r>0 .
$$

Then by (1.18), (4.37), (4.44), (4.51) and the fact $A_{1}+A_{2}=\bar{\sigma}-\nu \bar{\beta}$, we have

$$
\alpha_{0}(\gamma)=\bar{\sigma}-\hat{\sigma}-\nu \bar{\beta}-y_{0}\left(R_{s}\right)=A_{1} G_{0}\left(\sqrt{\lambda_{1}} R_{s}\right)+A_{2} G_{0}\left(\sqrt{\lambda_{2}} R_{s}\right)=R_{s} f^{\prime}\left(R_{s}\right) .
$$

Hence, assertion (i) holds. By (4.44), (4.54) and Lemma 4.4, we immediately obtain assertions (ii) and (iii). This completes the proof.

It is interesting to compare the above threshold value $\gamma_{*}$ with the corresponding threshold value obtained by $\mathrm{Wu}$ and Cui [27], which we denote by $\tilde{\gamma}_{*}$, for the tumor model with porous medium tissue. Recall that $\tilde{\gamma}_{*}=\sup \left\{0, \tilde{\gamma}_{2}, \tilde{\gamma}_{3} \cdots\right\}$, where (see (4.34) of [27])

$$
\tilde{\gamma}_{l}=\frac{2 R_{s}^{3}}{l(l+2)(l-1)}\left(\bar{\sigma}-\hat{\sigma}-\nu \bar{\beta}-y_{l}\left(R_{s}\right)\right) .
$$

We have the following result:

Lemma 4.8. (i) If $0<R_{s}<\sqrt{210} / 5$, then $\tilde{\gamma}_{*}<\gamma_{*}$.

(ii) If $R_{s}>0$ is large enough, then $\tilde{\gamma}_{*}>\gamma_{*}$. 
Proof. By (4.43) and (4.56), we have that, for $l \geq 2, \frac{\tilde{\gamma}_{l}}{\gamma_{l}}=\frac{(2 l+1) R_{s}^{2}}{2\left(l^{2}-1\right)(2 l+3)}$. Note that $\inf \left\{\frac{2\left(l^{2}-1\right)(2 l+3)}{2 l+1}, l \geq 2\right\}=42 / 5$. Thus if $0<R_{s}<\sqrt{210} / 5$, we have $0<\frac{\tilde{\gamma}_{l}}{\gamma_{l}}<1$. The desired results follow from Lemma 4.5 and the definition of $\gamma_{*}$ and $\tilde{\gamma}_{*}$.

\section{Asymptotic Behavior}

In this section we study asymptotic behavior of solutions of (1.1)-(1.11) and give the proof of Theorem 1.1.

It should be noted that the standard linearized stability theorem cannot be applied directly to problem (1.1)-(1.11), because 0 is an eigenvalue of $D \mathcal{Q}(0)$. We overcome this difficulty by employing Cui's local phase theorem for parabolic differential equation with invariance in Banach spaces (cf. Theorem 2.1 in [9] and [30]) and by constructing a local invariant center-stable manifold. To this end, for small $\epsilon>0$, we denote

$$
\mathbb{G}:=\left\{x \in \mathbb{R}^{3},|x|<\epsilon\right\} .
$$

Regarding $\mathbb{R}^{3}$ as a three-dimensional commutative Lie group, we see that $\mathbb{G}$ is its local Lie group containing the unit element 0 . Next, we introduce a Lie group action of $\mathbb{G}$ on $B_{q q}^{3-1 / q}\left(\mathbb{S}^{2}\right)$ similarly as in $[9$. Given $z \in \mathbb{G}$, define a translation $S_{z}: \mathbb{R}^{3} \rightarrow \mathbb{R}^{3}$ by

$$
S_{z}(x)=x+z, \quad \text { for } x \in \mathbb{R}^{3} .
$$

Let $a_{0}>0$ be sufficiently small. Given $3<q<+\infty$, we denote

$$
\mathcal{O}^{\prime}:=\left\{\rho \in B_{q q}^{3-1 / q}\left(\mathbb{S}^{2}\right),\|\rho\|_{C^{1}\left(\mathbb{S}^{2}\right)}<a_{0}\right\} .
$$

Note that $\mathcal{O} \subset \mathcal{O}^{\prime}$, where $\mathcal{O}$ is defined at the beginning of Section 2. It is easy to see that for any $\rho \in \mathcal{O}^{\prime}$, there exists a unique $\tilde{\rho} \in B_{q q}^{3-1 / q}\left(\mathbb{S}^{2}\right)$ such that the hypersurface $r=R_{s}+\tilde{\rho}(\omega)$ is the image of $S_{z}$ on $r=R_{s}+\rho(\omega)$, provided that $a_{0}$ and $\epsilon$ are sufficiently small. We define a mapping $\mathrm{S}_{z}^{*}: \mathcal{O}^{\prime} \rightarrow B_{q q}^{3-1 / q}\left(\mathbb{S}^{2}\right)$ by

$$
\mathrm{S}_{z}^{*}(\rho)=\tilde{\rho}
$$

Denote by $D \mathrm{~S}_{z}^{*}(\rho)$ and $D_{z} \mathrm{~S}_{z}^{*}(\rho)$ the Fréchet derivatives of $\mathrm{S}_{z}^{*}(\rho)$ in $\rho$ and $z$, respectively. By Lemma 4.3 of [9] we have the following results:

Lemma 5.1. Let $a_{0}>0$ and $\epsilon>0$ be sufficiently small. Then

(i) For any $z \in \mathbb{G}$, we have $\mathrm{S}_{z}^{*} \in C\left(\mathcal{O}^{\prime}, B_{q q}^{3-1 / q}\left(\mathbb{S}^{2}\right)\right) \cap C\left(\mathcal{O}, B_{q q}^{4-1 / q}\left(\mathbb{S}^{2}\right)\right)$.

(ii) For any $z, w \in \mathbb{G}$ we have

$$
\mathrm{S}_{z}^{*} \circ \mathrm{S}_{w}^{*}=\mathrm{S}_{z+w}^{*}, \quad \mathrm{~S}_{0}^{*}=i d \quad \text { and } \quad\left(\mathrm{S}_{z}^{*}\right)^{-1}=\mathrm{S}_{-z}^{*} .
$$

(iii) The mapping $\mathrm{S}^{*}: z \rightarrow \mathrm{S}_{z}^{*}$ from $\mathbb{G}$ to $C\left(\mathcal{O}^{\prime}, B_{q q}^{3-1 / q}\left(\mathbb{S}^{2}\right)\right)$ is an injection, and

$$
\mathrm{S}^{*} \in C^{k}\left(\mathbb{G}, C^{l}\left(\mathcal{O}, B_{q q}^{4-k-l-1 / q}\left(\mathbb{S}^{2}\right)\right)\right), \quad k \geq 0, \quad l \geq 0, \quad k+l \leq 3 .
$$

Moreover, for fixed $z \in \mathbb{G}$, there holds $D \mathrm{~S}_{z}^{*} \in C\left(\mathcal{O}, L\left(B_{q q}^{3-1 / q}\left(\mathbb{S}^{2}\right)\right)\right)$.

(iv) Define $p: \mathbb{G} \times \mathcal{O}^{\prime} \rightarrow B_{q q}^{3-1 / q}\left(\mathbb{S}^{2}\right)$ by $p(z, \rho):=\mathrm{S}_{z}^{*}(\rho)$ for $(z, \rho) \in \mathbb{G} \times \mathcal{O}^{\prime}$. Then for any $\rho \in \mathcal{O}$ we have $p(\cdot, \rho) \in C^{1}\left(\mathbb{G}, B_{q q}^{3-1 / q}\left(\mathbb{S}^{2}\right)\right)$, and $\operatorname{rank} D_{z} p(z, \rho)=3$ for every $z \in \mathbb{G}$ and $\rho \in \mathcal{O}$. If furthermore $\rho \in C^{\infty}\left(\mathbb{S}^{2}\right)$, then $p(\cdot, \rho) \in C^{\infty}\left(\mathbb{G}, C^{\infty}\left(\mathbb{S}^{2}\right)\right)$. 
It follows from Lemma 5.1 that $p$ is actually a local Lie group action of $\mathbb{G}$ on $B_{q q}^{3-1 / q}\left(\mathbb{S}^{2}\right)$ in the sense of 9 .

Lemma 5.2. Let $a_{0}>0$ and $\epsilon>0$ be sufficiently small. Then

(i) If $\rho$ is a solution of (3.10) with initial data $\rho_{0} \in \mathcal{O}$, then for any $z \in \mathbb{G}, \mathrm{S}_{z}^{*}(\rho)$ is also a solution of (3.10), with initial data $\mathrm{S}_{z}^{*}\left(\rho_{0}\right) \in \mathcal{O}$.

(ii) For any $z \in \mathbb{G}$ and $\rho \in \mathcal{O}$, there holds

$$
D \mathrm{~S}_{z}^{*}(\rho) \mathcal{Q}(\rho)=\mathcal{Q}\left(\mathrm{S}_{z}^{*}(\rho)\right) .
$$

Proof. (i) Since problem (1.1)-(1.11) and problem (3.10) are equivalent and (1.1)(1.11) is invariant under the coordinate translations, we immediately get assertion (i).

(ii) By Theorem 3.5, we have that for any $\rho_{0} \in \mathcal{O}$, there exists $T>0$ such that problem (3.10) has a unique strict solution $\rho\left(t, \rho_{0}\right) \in C([0, T], \mathcal{O}) \cap C^{1}([0, T]$, $\left.B_{q q}^{4-1 / q}\left(\mathbb{S}^{2}\right)\right)$. Denote $\tilde{\rho}(t)=\mathrm{S}_{z}^{*}\left(\rho\left(t, \rho_{0}\right)\right)$. Then assertion (i) implies that $\tilde{\rho}(t)$ is also a strict solution of (3.10) such that $\tilde{\rho}(0)=\mathrm{S}_{z}^{*}\left(\rho_{0}\right)$. Hence, we have

$$
\frac{\mathrm{d} \tilde{\rho}(t)}{\mathrm{d} t}=\mathcal{Q}(\tilde{\rho}(t)) \quad \text { for } 0<t \leq T \text {. }
$$

On the other hand,

$$
\begin{aligned}
\frac{\mathrm{d} \tilde{\rho}(t)}{\mathrm{d} t} & =\frac{\mathrm{dS}_{z}^{*}\left(\rho\left(t, \rho_{0}\right)\right)}{\mathrm{d} t}=D \mathrm{~S}_{z}^{*}\left(\rho\left(t, \rho_{0}\right)\right) \frac{\mathrm{d} \rho\left(t, \rho_{0}\right)}{\mathrm{d} t} \\
& =D \mathrm{~S}_{z}^{*}\left(\rho\left(t, \rho_{0}\right)\right) \mathcal{Q}\left(\rho\left(t, \rho_{0}\right)\right) \quad \text { for } 0<t \leq T .
\end{aligned}
$$

Thus

$$
D \mathrm{~S}_{z}^{*}\left(\rho\left(t, \rho_{0}\right)\right) \mathcal{Q}\left(\rho\left(t, \rho_{0}\right)\right)=\mathcal{Q}(\tilde{\rho}(t)) \quad \text { for } 0<t \leq T .
$$

Letting $t \rightarrow 0^{+}$, we get $D \mathrm{~S}_{z}^{*}\left(\rho_{0}\right) \mathcal{Q}\left(\rho_{0}\right)=\mathcal{Q}\left(\mathrm{S}_{z}^{*}\left(\rho_{0}\right)\right)$. Then (5.3) follows by the arbitrariness of $\rho_{0} \in \mathcal{O}$. The proof is completed.

Then we can give the proof of Theorem 1.1 by applying Theorem 2.1 of $[9,30$.

Proof of Theorem 1.1. (i) Noting

$$
-D \mathcal{Q}(0) \in \mathcal{H}\left(B_{q q}^{4-1 / q}\left(\mathbb{S}^{2}\right), B_{q q}^{3-1 / q}\left(\mathbb{S}^{2}\right)\right),
$$

by Lemma 3.4 we know that $D \mathcal{Q}(0)$ is a sectorial operator in $B_{q q}^{3-1 / q}\left(\mathbb{S}^{2}\right)$ with domain $B_{q q}^{4-1 / q}\left(\mathbb{S}^{2}\right)$, and its graph norm is equivalent to the norm of $B_{q q}^{4-1 / q}\left(\mathbb{S}^{2}\right)$. Thus the assumption $\left(B_{1}\right)$ of Theorem 2.1 of $[9$ is satisfied for the operator $D \mathcal{Q}(0)$. By Lemma 5.1 and Lemma 5.2, we see that equation (3.10) is quasi-invariant under the Lie group action $(\mathbb{G}, p)$, so the assumption $\left(B_{2}\right)$ of Theorem 2.1 of $[9$ ] follows. Define $X_{0}:=\operatorname{span}\left\{Y_{1 m}(\omega), m=-1,0,1\right\}$ and

$$
X_{1}:=\left\{\rho \in B_{q q}^{3-1 / q}\left(\mathbb{S}^{2}\right): \rho \text { is orthogonal to } X_{0} \text { in } L^{2}\left(\mathbb{S}^{2}\right)\right\} .
$$

It is easy to see that $X_{1}$ is closed in $B_{q q}^{3-1 / q}\left(\mathbb{S}^{2}\right)$ and $B_{q q}^{3-1 / q}\left(\mathbb{S}^{2}\right)=X_{0} \oplus X_{1}$. For any $\gamma>0$ and $\gamma \notin\left\{\gamma_{l}\right\}_{l \geq 2}$, it follows from Lemma 4.3, Lemma 4.4 and a standard argument that $\operatorname{Ker} D \mathcal{Q}(0)=X_{0}$ and Range $D \mathcal{Q}(0)=X_{1}$.

Note that $\gamma_{*}=\sup \left\{0, \gamma_{l}, l \geq 2\right\}$. If $f^{\prime}\left(R_{s}\right)<0$ and $\gamma>\gamma_{*}$, then we get

$$
\operatorname{dimKer} D \mathcal{Q}(0)=3=\operatorname{rank} D_{z} p(z, \rho) \quad \text { for } z \in \mathbb{G}, \rho \in \mathcal{O} \text {. }
$$


Thus the assumption $\left(B_{3}\right)$ of Theorem 2.1 of 9 holds. Moreover, from Lemma 4.4 and Lemma 4.7 we have

$$
\omega_{-}:=-\sup \{\operatorname{Re} \lambda: \lambda \in \sigma(D \mathcal{Q}(0)) \backslash\{0\}\}>0,
$$

which implies that assumption $\left(B_{4}\right)$ of Theorem 2.1 of $[9]$ is satisfied for $D \mathcal{Q}(0)$. Hence, by applying Theorem 2.1 of 9 , we get that there exist two neighborhoods $\mathcal{O}_{1}$ and $\mathcal{O}_{2}, \mathcal{O}_{2} \subset \mathcal{O}_{1} \subset \mathcal{O}$ such that for each $\rho_{0} \in \mathcal{O}_{2}$ the Cauchy problem (3.10) has a unique global solution

$$
\rho:=\rho\left(t, \rho_{0}\right) \in C\left([0, \infty), \mathcal{O}_{1}\right) \cap C^{1}\left([0, \infty), B_{q q}^{3-1 / q}\left(\mathbb{S}^{2}\right)\right),
$$

and the center manifold is given by

$$
\mathcal{M}^{c}=\left\{\mathrm{S}_{z}^{*}(0), z \in \mathbb{G}\right\} \cap \mathcal{O}_{1},
$$

which is a three-dimensional smooth manifold consisting of all stationary solutions and attracting all small global solutions of (3.10) exponentially. Moreover, for any $\rho_{0} \in \mathcal{O}_{2}$ and any $0<\omega<\omega_{-}$, there exist a unique couple $\left(x_{0}, \tilde{\rho}_{0}\right) \in \mathbb{G} \times \mathcal{O}_{1}$ and a positive constant $K=K(\omega)$ such that $\rho_{0}=\mathrm{S}_{x_{0}}^{*}\left(\tilde{\rho}_{0}\right)$ and

$$
\begin{aligned}
& \left\|\rho\left(t ; \tilde{\rho}_{0}\right)-0\right\|_{B_{q q}^{4-1 / q}\left(\mathbb{S}^{2}\right)} \leq K \mathrm{e}^{-\omega t}, \\
& \left\|\rho\left(t ; \rho_{0}\right)-\mathrm{S}_{x_{0}}^{*}(0)\right\|_{B_{q q}^{4-1 / q}\left(\mathbb{S}^{2}\right)} \leq K \mathrm{e}^{-\omega t} .
\end{aligned}
$$

Noting the notation $0_{\left[x_{0}\right]}$ introduced in Section 1 , we have $0_{\left[x_{0}\right]}=\mathrm{S}_{x_{0}}^{*}(0)$ for any $x_{0} \in \mathbb{G}$. Thus (1.21) follows from Lemma 2.1 and Lemma 3.3.

(ii) Assume now that $f^{\prime}\left(R_{s}\right)>0$ or $0<\gamma<\gamma_{*}$. Then by Lemma 4.3 and Lemma 4.7 (iii), we have

$$
\sigma_{+}(D \mathcal{Q}(0)) \neq \emptyset \quad \text { and } \quad \sigma(D \mathcal{Q}(0))=\sigma_{-}(D \mathcal{Q}(0)) \cup\{0\} \cup \sigma_{+}(D \mathcal{Q}(0))
$$

and

$$
-\omega_{-}:=\sup _{\lambda \in \sigma_{-}(D \mathcal{Q}(0))}\{\operatorname{Re} \lambda\}<0<\omega_{+}:=\inf _{\lambda \in \sigma_{+}(D \mathcal{Q}(0))}\{\operatorname{Re} \lambda\} .
$$

It follows that assumption $\left(B_{4}\right)$ of Theorem 2.1 of 30 is satisfied. By Theorem 9.1.3 in [25] we know that the zero equilibrium of the Cauchy problem (3.10) is unstable. Thus by the equivalence of the problem (3.10) and problem (1.1)-(1.11), we have that $\left(\sigma_{s}, \beta_{s}, \mathbf{v}_{s}, p_{s}, 0\right)$ is unstable.

Define the spectral projection associated to $D \mathcal{Q}(0)$ by

$$
P:=\frac{1}{2 \pi i} \int_{\Gamma}(\lambda-D \mathcal{Q}(0))^{-1} \mathrm{~d} \lambda
$$

where $\Gamma$ is a closed smooth curve in the complex plane enclosing the spectral part $\{0\} \cup \sigma_{+}(D \mathcal{Q}(0))$.

If furthermore $\gamma \notin\left\{\gamma_{l}\right\}_{l \geq 2}$, similarly as above, we can easily verify that assumptions $\left(B_{1}\right)-\left(B_{3}\right)$ of Theorem 2.1 of [30, which are the same as the ones of Theorem 2.1 of [9], are satisfied. Hence, by applying Theorem 2.1 (i) of [30] and (3.9), we have that there exist a neighborhood $O_{0} \subset \mathcal{O}$ and a smooth mapping

$$
\Re: O_{0} \cap(I-P) B_{q q}^{4-1 / q}\left(\mathbb{S}^{2}\right) \rightarrow P\left(B_{q q}^{4-1 / q}\left(\mathbb{S}^{2}\right)\right),
$$

with $\mathfrak{R}^{\prime}(0)=0$, such that $\mathcal{M}:=\operatorname{Graph}(\mathfrak{R})$ constructs a center-stable manifold of (3.10) having the following properties: For any $\rho_{0} \in O_{0}$ satisfying $\rho_{0} \in \operatorname{Graph}(\mathfrak{R})$, the solution $\rho\left(t, \rho_{0}\right)$ of $(3.10)$ exists globally and converges to the zero equilibrium exponentially; conversely, if the global solution $\rho\left(t, \rho_{0}\right)$ of (3.10) converges to the zero equilibrium exponentially and $\rho_{0} \in O_{0} \cap(I-P) B_{q q}^{4-1 / q}\left(\mathbb{S}^{2}\right)$, then 
$\rho_{0} \in \operatorname{Graph}(\Re)$. Besides, by Theorem 2.1 (iii) of [30, there exist two neighborhoods $O_{2} \subset O_{1} \subset O_{0}$ such that for any $\rho_{0} \in O_{2}$, if the solution $\rho\left(t, \rho_{0}\right)$ exists globally and is contained in $O_{1}$, i.e., $\rho\left(t, \rho_{0}\right) \in C\left([0, \infty), O_{1}\right)$; then there exists a unique couple $\left(x_{0}, \tilde{\rho}_{0}\right) \in \mathbb{G} \times \operatorname{Graph}(\Re)$ such that $\rho_{0}=\mathrm{S}_{x_{0}}^{*}\left(\tilde{\rho}_{0}\right)$ and (5.6) holds for all $0<\omega<\omega_{-}$and some positive constant $K=K(\omega)$. Moreover, by Lemma 5.2, for the solution with initial data $\tilde{\rho}_{0}$, we have $\rho\left(t, \tilde{\rho}_{0}\right)=\mathrm{S}_{x_{0}}^{*}\left(\rho\left(t, \rho_{0}\right)\right)=\rho_{\left[x_{0}\right]}\left(t, \rho_{0}\right)$, then there holds $\rho_{\left[x_{0}\right]}\left(t, \rho_{0}\right) \in \operatorname{Graph}(\mathfrak{R})$. This completes the proof of Theorem 1.1 .

\section{Conclusions And Biological implications}

We have studied a free boundary problem modeling the growth of tumors with fluid-like tissue under the action of inhibitors. By first transforming the moving domain into a fixed reference domain we convert the free boundary problem into a Cauchy problem in Banach spaces. Then by analyzing the linearized problem at the radial stationary solution and using analytic semigroup theory and Cui's local phase theorem $([9,30])$, we proved that if the radial stationary solution is asymptotically stable under small radial perturbations, then there exists a nonnegative threshold value $\gamma_{*}$ such that if $\gamma>\gamma_{*}$, then it is still asymptotically stable under small non-radial perturbations, whereas if $0<\gamma<\gamma_{*}$, then it is unstable, and we also give a description of the local phase of unstable solutions.

From the point of view of modeling, the mathematical results give us some interesting biological implications. Instability of a tumor means that the tumor may change shape and, in particular, may develop protrusions or "fingers" into the surrounding tissue and become invasive. The parameter $\gamma$ is the cell-to-cell adhesiveness and plays an important role in keeping the tumor cohesive ([4, 5]); tumor invasion into neighborhood tissue is associated with decreased cell-to-cell adhesiveness. To assess the effect of inhibitors on tumor's growth, we show that the threshold value $\gamma_{*}$ is a monotone decreasing function of the inhibitor supply $\bar{\beta}$. This implies that an increasing inhibitor supply may increase the probability of tumor growth evolving into a dormant state. Thus, the inhibitor has a positive effect on the tumor's treatment, promotes the tumor's stability and makes it benign.

The material properties of tumor tissue and its surroundings also effect the stability of tumor growth $([18,19])$. It is interesting to compare a tumor with fluidlike tissue and a solid tumor with porous medium tissue. This can be achieved by comparing the threshold value $\gamma_{*}$ with the corresponding one, which we denote by $\tilde{\gamma}_{*}$, obtained in [27. for a solid tumor with porous medium tissue modeled by Darcy's law. The result shows that if $0<R_{s}<\sqrt{210} / 5$, then $\gamma_{*}>\tilde{\gamma}_{*}$, while $\gamma_{*}<\tilde{\gamma}_{*}$ if $R_{s}$ is large enough. Thus, a tumor with fluid-like tissue has its own characteristics. It indicates that a tumor with fluid-like tissue is more hazardous than a solid tumor with porous medium tissue for small $R_{s}$, whereas for large enough $R_{s}$ this is reversed, that is, a tumor with fluid-like tissue is more unaggressive.

These results are hoped to be useful in scientific studies of mechanisms of tumor growth and the medical treatment of cancers.

\section{ACKNOWLEDGEMENT}

The authors would like to thank the anonymous referee for valuable suggestions. 


\section{REFERENCES}

1. H. Amann, Linear and Quasilinear Parabolic Problems, Vol. I, Birkhäuser, Basel, 1995. MR:1345385 (96g:34088)

2. B. Bazaliy and A. Friedman, Global existence and asymptotic stability for an ellipticparabolic free boundary problem: An application to a model of tumor growth, Indiana Univ. Math. J., 52 (2003), 1265-1304. MR2010327 (2004j:35302)

3. H. M. Byrne, A weakly nonlinear analysis of a model of avascular solid tumor growth, J. Math. Biol., 39 (1999), 59-89. MR1705626(2000i:92011)

4. H. M. Byrne and M. A. J. Chaplain, Growth of nonnecrotic tumors in the presence and absence of inhibitors, Math. Biosci., 130 (1995), 151-181.

5. H. M. Byrne and M. A. J. Chaplain, Growth of necrotic tumors in the presence and absence of inhibitors, Math. Biosci., 135 (1996), 187-216.

6. X. Chen, S. Cui and A. Friedman, A hyperbolic free boundary problem modeling tumor growth: Asymptotic behavior, Trans. Amer. Math. Soc., 357 (2005), 4771-4804. MR2165387 (2006d:34144)

7. S. Cui, Analysis of a mathematical model for the growth of tumors under the action of external inhibitors, J. Math. Biol., 44 (2002), 395-426. MR.1908130 (2003f:92019)

8. S. Cui, Well-posedness of a multidimensional free boundary problem modelling the growth of nonnecrotic tumors, J. Funct. Anal., 245 (2007), 1-18. MR2310801 (2009d:35349)

9. S. Cui, Lie group action and stability analysis of stationary solutions for a free boundary problem modelling tumor growth, J. Differential Equations, 246 (2009), 1845-1882. MR.2494690 (2010e:35305)

10. S. Cui and J. Escher, Asymptotic behaviour of solutions of a multidimensional moving boundary problem modeling tumor growth, Comm. Partial Differential Equations, 33 (2008), 636655. MR2424371 (2009k:35331)

11. J. Escher, Classical solutions to a moving boundary problem for an elliptic-parabolic system, Interfaces Free Bound., 6 (2004), 175-193. MR2079602 (2006a:35316)

12. S. J. H. Franks, H. M. Byrne, J. P. King, J. C. E. Underwood and C. E. Lewis, Modelling the early growth of ductal carcinoma in situ of the breast, J. Math. Biol., 47 (2003), 424-452. MR2029006 (2004j:92030)

13. S. J. H. Franks, H. M. Byrne, J. P. King, J. C. E. Underwood and C. E. Lewis, Modelling the growth of comedo ductal carcinoma in situ, Math. Med. Biol., 20 (2003), 277-308.

14. S. J. H. Franks, H. M. Byrne, J. C. E. Underwood and C. E. Lewis, Biological inferences from a mathematical model of comedo ductal carcinoma in situ of the breast, J. Theoret. Biol., 232 (2005), 523-543. MR2125830

15. S. J. H. Franks and J. P. King, Interactions between a uniformly proliferating tumour and its surroundings: Uniform material properties, Math. Med. Biol., 20 (2003), 47-89.

16. A. Friedman, A free boundary problem for a coupled system of elliptic, hyperbolic and Stokes equations modeling tumor growth, Interfaces Free Bound., 8 (2006), 247-261. MR2256843 (2007e:35299)

17. A. Friedman, Mathematical analysis and challenges arising from models of tumor growth, Math. Models Methods Appl. Sci., 17, suppl. (2007), 1751-1772. MR2362763 (2009d:92028)

18. A. Friedman and B. Hu, Bifurcation for a free boundary problem modeling tumor growth by Stokes equation, SIAM J. Math. Anal., 39 (2007), 174-194. MR2318381 (2008f:35407)

19. A. Friedman and B. Hu, Bifurcation from stability to instability for a free boundary problem modeling tumor growth by Stokes equation, J. Math. Anal. Appl., 327 (2007), 643-664. MR.2277439 (2008e:35213)

20. A. Friedman and F. Reitich, Analysis of a mathematical model for growth of tumor, J. Math. Biol., 38 (1999), 262-284. MR1684873(2001f:92011)

21. A. Friedman and F. Reitich, Symmetry-breaking bifurcation of analytic solutions to free boundary problems: An application to a model of tumor growth, Trans. Amer. Math. Soc., 353 (2001), 1587-1634. MR1806728(2002a:35208)

22. H. P. Greenspan, On the growth of cell culture and solid tumors, J. Theoret. Biol., 56 (1976), 229-242. MR0429164 (55:2183)

23. E. L. Hill, The theory of vector spherical harmonics, Amer. J. Phys., 222 (1954), 211-214. MR0061226 (15:792h) 
24. J. S. Lowengrub, H. B. Frieboes, F. Jin, et al., Nonlinear modelling of cancer: Bridging the gap between cells and tumours, Nonlinearity, 23 (2010), R1-R91. MR2576370

25. A. Lunardi, Analytic Semigroups and Optimal Regularity in Parabolic Problems, Birkhäuser, Basel, 1995. MR1329547 (96e:47039)

26. G. N. Watson, A Treatise on the Theory of Bessel Functions, Cambridge University Press, Cambridge, 1944. MR0010746 (6:64a)

27. J. Wu and S. Cui, Asymptotic behaviour of solutions of a free boundary problem modelling the growth of tumours in the presence of inhibitors, Nonlinearity, 20 (2007), 2389-2408. MR.2356116 (2009b:35446)

28. J. Wu and S. Cui, Asymptotic behavior of solutions of a free boundary problem modeling the growth of tumors with Stokes equations, Discrete Contin. Dyn. Syst., 24 (2009), 625-651. MR2486594 (2010g:35349)

29. J. Wu and S. Cui, Asymptotic stability of stationary solutions of a free boundary problem modeling the growth of tumors with fluid tissues, SIAM J. Math. Anal., 41 (2009), 391-414. MR:2505864 (2010m:35578)

30. J. Wu and S. Cui, Asymptotic behavior of solutions for parabolic differential equations with invariance and applications to a free boundary problem modeling tumor growth, Discrete Contin. Dyn. Syst., 26 (2010), 737-765. MR2556506(2011d:35528)

31. F. Zhou and S. Cui, Well-posedness and stability of a multidimentional moving boundary problem modeling the growth of tumor cord, Discrete Contin. Dyn. Syst., 21 (2008), 929-943. MR2399443 (2009c:35487)

32. F. Zhou and J. Wu, Regularity of solutions to a free boundary problem modeling tumor growth by Stokes equation, J. Math. Anal. Appl., 377 (2011), 540-556. MR2769156

Department of Mathematics, Soochow University, Suzhou, Jiangsu 215006, People's RePublic OF CHINA

E-mail address: wjdmath@yahoo.com.cn

Department of Mathematics, South China University of Technology, Guangzhou, Guangdong 510640, People's Republic of China

E-mail address: zhoufujunht@yahoo.com.cn 